\title{
Comparing faceted and smoothed tool surface descriptions in sheet metal forming simulation
}

\author{
D. M. Neto • M. C. Oliveira $・$ J. L. Alves $・$ L. F. Menezes
}

Received: 30 December 2013 / Accepted: 4 May 2014 /Published online: 24 May 2014

(C) Springer-Verlag France 2014

\begin{abstract}
This study deals with different tool surface description methods used in the finite element analysis of sheet metal forming processes. The description of arbitrarily-shaped tool surfaces using the traditional linear finite elements is compared with two distinct smooth surface description approaches: (i) Bézier patches obtained from the ComputerAided Design model and (ii) smoothing the finite element mesh using Nagata patches. The contact search algorithm is presented for each approach, exploiting its special features in order to ensure an accurate and efficient contact detection. The influence of the tool modelling accuracy on the numerical results is analysed using two sheet forming examples, the unconstrained cylindrical bending and the reverse deep drawing of a cylindrical cup. Smoothing the contact surfaces with Nagata patches allows creating more accurate tool models, both in terms of shape and normal vectors, when compared with the conventional linear finite element mesh. The computational efficiency is evaluated in this study through the total number of increments and the required CPU time. The mesh refinement in the faceted description approach is not effective in terms of computational efficiency due to large discontinuities in the normal vector field across facets, even when adopting fine meshes.
\end{abstract}

Keywords Sheet metal forming · Finite element method . Contact mechanics $\cdot$ Tool surface description $\cdot$ Surface smoothing

D. M. Neto $(\bowtie) \cdot$ M. C. Oliveira $\cdot$ L. F. Menezes

CEMUC Department of Mechanical Engineering, University of

Coimbra, Polo II Rua Luís Reis Santos, Coimbra, Pinhal de

Marrocos 3030-788, Portugal

e-mail: diogo.neto@dem.uc.pt

J. L. Alves

CT2M Department of Mechanical Engineering, University of

Minho, Campus de Azurém, Guimarães 4800-058, Portugal

\section{Introduction}

The strong reduction of development periods observed in the automotive industry, which is imposed by the powerful global competition, led to the redefinition of the conventional manufacturing procedures used in sheet metal forming process [1]. Thus, the traditional tool design methods based on a trial-and-error loops or empirical procedures have been progressively substituted by means of finite element analysis, shortening the delivery periods [2]. Nowadays, through the simultaneous improvement of solving methods and computer technology (e.g. multi-core processors), the numerical simulation of 3D sheet metal forming process can be used to predict the occurrence of some defects, such as fracture, necking, wrinkling and springback. [3, 4]. Hence, in order to improve the accuracy of sheet metal forming simulation, numerous efforts have been made in the last decade, which can be divided in in two categories: (i) development of new finite elements [5-7] and (ii) development of new material constitutive models $[8,9]$. Nevertheless, the mechanical modelling of sheet metal forming comprises three strong nonlinearities, which are dictated by the frictional contact, the geometrical nonlinearity produced by large deformation and the material nonlinearity due to the elastoplastic behaviour [10]. In fact, the numerical modelling of the contact between the sheet and tools plays an important role in the material deformation, which is directly associated with the tool modelling accuracy [11-13].

Typically, the forming tools can be considered rigid in the finite element model, allowing to model only their outer surfaces. Therefore, in sheet metal forming processes the contact occurs between a deformable body (sheet) and several rigid obstacles (tools), which represents a relatively simple case of contact between deformable bodies [4]. Taking into account this simplification, the tool surfaces geometry is commonly defined by using one of the three following different 
approaches: (i) analytical functions, (ii) finite elements or (iii) parametric patches. The first approach is restricted to simple tool geometries, since the geometry is composed by an assembly of simple analytical shapes [12]. On the other hand, for arbitrarily-shaped tool surfaces, the finite element mesh approach is usually employed due to its ability and simplicity. However, this approximation can lead to large errors in the geometry, requiring an extremely finer mesh in curved regions to obtain an accurate tool description $[12,14]$. The last approach resorts to parametric patches, which can be obtained either directly from the Computer-Aided Design (CAD) model or by smoothing the finite element mesh. Concerning the first option, trimmed NURBS patches are commonly adopted in CAD systems, dictating very complex and computationally expensive algorithms to deal with these patches [15]. Furthermore, CAD models are known to be plagued by geometrical or topological errors and inconsistencies [16], which make its application difficult within a finite element environment. On the other hand, several surface smoothing techniques, such as NURBS [17], Bézier [18] and Gregory patch [19], have been proposed to overcome the problems related with the accuracy of the finite element mesh description. Indeed, the faceted tool surface description, resulting from the spatial discretization of the contact surfaces with linear finite elements, leads to artificial oscillations in the contact forces due to the discontinuous contact surface normal field, which causes convergence problems in the iterative procedure $[13,14]$. In order to overcome this chattering effect, new techniques based on mortar methods have been successfully introduced in computational contact mechanics [20]. Using this approach, the contact constraints are imposed in an integral form instead of nodal enforcement, commonly used in the classical node-to-segment contact formulations. Thus, the mortar approach provides superior robustness in the solution of contact problems involving large sliding due to the integral evaluation of the contact conditions. This is particularly effective for faceted contact surfaces, where the integral evaluation of the surface contribution produces an artificial smoothing $[21,22]$. More recently, the isogeometric analysis was introduced for the numerical simulation of frictional contact problems, providing a more accurate geometric representation than traditional finite elements [23].

The objective of the present study is to analyse the effect of tool surface modelling accuracy on the numerical solution of sheet metal forming processes simulation. Three different methods to describe the forming tools are presented: (i) faceted surface description using bilinear finite elements, (ii) smoothing the finite element mesh with Nagata patches and (iii) applying Bézier patches obtained from CAD. The geometrical accuracy achieved in the tool surface definition, using these different surface description methods, is evaluated and compared. Besides, the contact search algorithm used by each surface description method is described, since it is highly dependent of the selected description method. Two distinct examples of sheet metal forming are carried out to compare the effectiveness of the presented methods. The selected numerical examples comprise the unconstrained cylindrical bending and the reverse deep drawing process of a cylindrical cup. The studied results take into account both the main process parameters and the numerical variables related with the contact between the sheet and the forming tools.

\section{Tool surface description methods}

The three principal tool surface description methods adopted in the numerical simulation of sheet metal forming processes, involving arbitrarily-shaped tools, are described in detail in this section. The first approach uses Bézier patches in the surface description, which are obtained with the aid of a specific CAD package. In the second approach, the surface finite element mesh is smoothed with Nagata patches, allowing an accurate approximation of the tool shape. Finally, the classical approach is presented, which describes the tool surfaces through a finite element mesh using bilinear finite elements. All these approaches were implemented in DD3IMP finite element code, which has been specifically developed to simulate sheet metal forming processes [10]. Its main characteristic is the use of a fully implicit algorithm of Newton-Raphson type to solve, within a single iterative loop, the non-linearities related with the frictional contact problem and the elastoplastic behaviour of the deformable body. The frictional contact is defined by the Coulomb law and treated with a mixed formulation, using the augmented Lagrangian approach proposed by Alart and Curnier [24]. In order to analyse medium scale problems in an acceptable computational time, some high performance computing techniques have been incorporated, such as OpenMP directives [25].

\section{Bézier patches}

Typically, the geometric definition of the surfaces composing the forming tools is performed with the aid of CAD software packages. Thus, the information about the Bézier patches can be extracted directly from some CAD packages using the standard STEP file format. However, this strategy involves some limitations due to the inherent simplicity of Bézier patches, particularly when applied to describe very complex geometries. Indeed, it is necessary to perform some careful operations on the CAD model, such as surfaces division, in order to attain a proper geometrical definition with patches presenting a reduced degree. Nevertheless, this laborious manual intervention process is incompatible with complex models involving hundreds of patches [26].

The most widely used method in geometric modelling applications is the tensor product scheme, which is basically 
a bidirectional curve scheme [27]. Thus, a Bézier patch can be defined as the tensorial product of two Bézier curves, given by:

$\mathbf{S}(\eta, \zeta)=\sum_{i=0}^{n} \sum_{j=0}^{m} B_{i, n}(\eta) B_{j, m}(\zeta) \mathbf{P}_{i j}, \quad$ with $\quad 0 \leq \eta, \zeta \leq 1$,

where $\mathbf{P}_{i j}$ are the position vector of the vertices composing the polygonal control net, leading to a total of $(n+1)(m+1)$ control points, since the indices $n$ and $m$ present a variation equal to the number of polygon vertices less one, in each principal direction of the patch. The $B_{i, n}$ and $B_{j, m}$ are the Bernstein basis functions, defined in each direction through the local coordinates $\eta$ and $\zeta$. The Bernstein polynomial of degree $n$ is defined by:

$B_{i, n}(t)=\left(\begin{array}{c}n \\ i\end{array}\right) t^{i}(1-t)^{n-i}, \quad$ for $\quad i=0,1, \ldots, n$,

where the binomial coefficient is given explicitly by

$$
\left(\begin{array}{c}
n \\
i
\end{array}\right)=\left\{\begin{array}{cc}
\frac{n !}{i !(n-i) !} & \text { if } \quad 0 \leq i \leq n \\
0 & \text { otherwise }
\end{array}\right.
$$

where $n$ ! denotes the factorial of $n$. In order to highlight the position of the control points that define the patch, Fig. 1 presents both the polygonal control net and the resulting Bézier patch.

Bézier patches can also be described using the monomial form, also called power basis form [28]. The main advantage of this form is that insures lower computational time for operations such as calculations of point coordinates or derivatives. However, it is numerically less stable, mainly for patches with high degree [28]. Taking into account its features, the power basis form is the one used in this study to describe the tool surfaces, where the maximum patch degree allowed in each parametric direction is limited to six to avoid numerical instabilities [26].

\section{Nagata patches}

The smoothing method selected in this study to improve the geometric accuracy of the tool surfaces is the one proposed by Neto et al. [29], where the contact surface is defined by Nagata patches. The key point of this method is the application of the Nagata patch interpolation algorithm [30], which recovers the curvature of the surfaces with good accuracy, using only the position and normal vectors at the finite element mesh vertices. The interpolation degree defining the Nagata patch is quadratic in both parametric directions and the patch typology is arbitrary, nevertheless in this study only triangular and quadrilateral patches are used (see Fig. 2). The first step of this strategy is performing the discretization of the tool surfaces using any mesh generator, producing a mesh that can be composed either by triangular or quadrilateral finite elements. Then, the required surface normal vectors in each vertex of the tool surface mesh are evaluated from the information available in the IGES file format [31], which was originally used in the surface mesh generation.

Considering the simplest case of an edge interpolation with a Nagata curve, the resulting curve is defined by:

$\mathbf{C}(\eta)=\mathbf{x}_{0}+\left(\mathbf{x}_{1}-\mathbf{x}_{0}-\mathbf{c}\right) \eta+\mathbf{c} \eta^{2}, \quad$ with $\quad 0 \leq \eta \leq 1$,

where $\eta$ is the local coordinate of the Nagata curve. The position vector of each edge end point is defined by $\mathbf{x}_{0}$ and $\mathbf{x}_{1}$, while its unit normal vectors are given by $\mathbf{n}_{0}$ and $\mathbf{n}_{1}$, respectively. The coefficient $\mathbf{c}$, which adds the curvature to the edge is calculated as:

$\mathbf{c}\left(\mathbf{x}_{0}, \mathbf{x}_{1}, \mathbf{n}_{0}, \mathbf{n}_{1}\right)=\left\{\begin{array}{ll}\frac{\left[\mathbf{n}_{0}, \mathbf{n}_{1}\right]}{1-a^{2}}\left[\begin{array}{cc}1 & -a \\ -a & 1\end{array}\right]\left\{\begin{array}{l}\mathbf{n}_{0} \cdot\left(\mathbf{x}_{1}-\mathbf{x}_{0}\right) \\ -\mathbf{n}_{1} \cdot\left(\mathbf{x}_{1}-\mathbf{x}_{0}\right)\end{array}\right\} & (a \neq \pm 1) \\ \frac{\left[\mathbf{n}_{0}, \pm \mathbf{n}_{0}\right]}{2}\left\{\begin{array}{l}\mathbf{n}_{0} \cdot\left(\mathbf{x}_{1}-\mathbf{x}_{0}\right) \\ \mp \mathbf{n}_{0} \cdot\left(\mathbf{x}_{1}-\mathbf{x}_{0}\right)\end{array}\right\}=\mathbf{0} & (a= \pm 1)\end{array}\right.$,

where $a=\mathbf{n}_{0} \cdot \mathbf{n}_{1}$ is the cosine of the angle between the normal vectors. The above formulation, described for an edge, can be extended to general polygonal patches, such as triangular and quadrilateral patches, assuring $G^{1}$ continuity at the vertices and quasi- $G^{1}$ across the edges between patches. The idea is to first interpolate independently each edge through the quadratic curve using Eqs. (4) and (5). Afterwards, the Nagata patch is defined by its trace on the quadratic segments [30].

In the case of a triangular finite element, schematically presented in Fig. 2 (a), the resulting Nagata patch is defined by the following quadratic polynomial:

$\mathbf{S}(\eta, \zeta)=\mathbf{c}_{00}+\mathbf{c}_{10} \eta+\mathbf{c}_{01} \zeta+\mathbf{c}_{11} \eta \zeta+\mathbf{c}_{20} \eta^{2}+\mathbf{c}_{02} \zeta^{2}, \quad$ with $\quad 0 \leq \zeta \leq \eta \leq 1$,

where the six coefficient vectors $\mathbf{c}_{i j}$ are calculated using only the position $\left(\mathbf{x}_{00}, \mathbf{x}_{10}\right.$ and $\left.\mathbf{x}_{11}\right)$ and normal vectors $\left(\mathbf{n}_{00}, \mathbf{n}_{10}\right.$ and $\mathbf{n}_{11}$ ) of the finite element vertices, following the relationships presented in $[30,32]$. The quadrilateral patch, exemplified in Fig. 2 (b), is interpolated in a similar way as the triangular patch. Hence, the quadrilateral Nagata patch is given by:

$$
\begin{aligned}
\mathbf{S}(\eta, \zeta)=\mathbf{c}_{00} & +\mathbf{c}_{10} \eta+\mathbf{c}_{01} \zeta+\mathbf{c}_{11} \eta \zeta+\mathbf{c}_{20} \eta^{2}+\mathbf{c}_{02} \zeta^{2}+\mathbf{c}_{21} \eta^{2} \zeta \\
& +\mathbf{c}_{12} \eta \zeta^{2}, \quad \text { with } \quad 0 \leq \eta, \zeta \leq 1,
\end{aligned}
$$

where the eight coefficient vectors $\mathbf{c}_{i j}$ are evaluated using the position $\left(\mathbf{x}_{00}, \mathbf{x}_{10}, \mathbf{x}_{11}\right.$ and $\left.\mathbf{x}_{01}\right)$ and normal vectors ( $\mathbf{n}_{00}, \mathbf{n}_{10}$, $\mathbf{n}_{11}$ and $\mathbf{n}_{01}$ ) of the finite element vertices through the relationships defined in $[30,32]$. Note that the vertices defining the finite element do not need to be coplanar. 
Fig. 1 Example of a Bézier patch: (a) the vertices composing the polygonal control net definition; (b) the resulting patch [27]

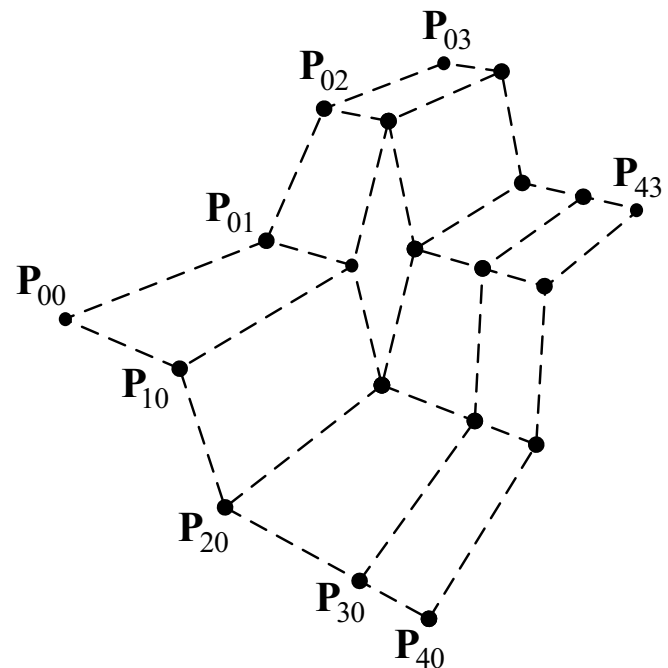

(a)

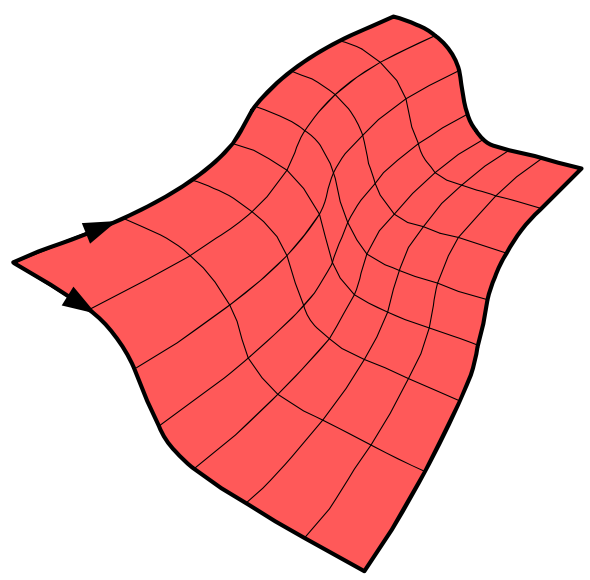

(b)
Bilinear facets

The traditional approach to model the tool surfaces, which uses bilinear finite elements to describe the contact surfaces, can be considered as a variant of the smoothing method without recovering the curvature. In fact, in this study the bilinear facets are defined through the Nagata patch interpolation, imposing zero value to the coefficient $\mathbf{c}$ defined in Eq. (5), which adds the curvature to the patch. This strategy allows comparing both tool surface description methods in a simple way, keeping the same discretization of the tool surfaces (number of facets/patches).

\section{Interpolation methods accuracy}

In order to highlight the geometrical improvements attained with the Nagata smoothing method over the faceted description, a 2D simple example is selected to assess the accuracy of the shape and the orientation of the normal vector. This section compares briefly the accuracy of the Nagata patch interpolation with the linear interpolation, using a circular arc. Two parameters are considered: (i) the radial error $\delta_{\mathrm{r}}$, which represents the distance between the interpolated curve and the perfect circle, measured in the radial direction and (ii) the normal vector orientation error $\delta_{\mathrm{n}}$, which stands for difference between the direction of the normal vector obtained with the interpolation curve and the direction of the normal vector to the perfect circle.

Figure 3 (a) presents the radial error distribution in a circular arc with a central angle of $30^{\circ}$ described by a single curve, comparing both the linear and the Nagata interpolation methods. Although the evolution of the radial error is roughly similar for both interpolation methods, the maximum value attained is completely different, which is located at the midpoint of the curve. Note that the figure presents two different scales since the order of magnitude in the results is not comparable. It is about $3.5 \%$ (inside the circular arc) in the linear interpolation, while the Nagata interpolation presents a maximum of only $0.06 \%$ (outside the circular arc). The distribution of the error in the normal vector orientation is presented in Fig. 3 (b) for the same circular arc. The

Fig. 2 Nagata patch interpolation: (a) triangular patch; (b) quadrilateral patch

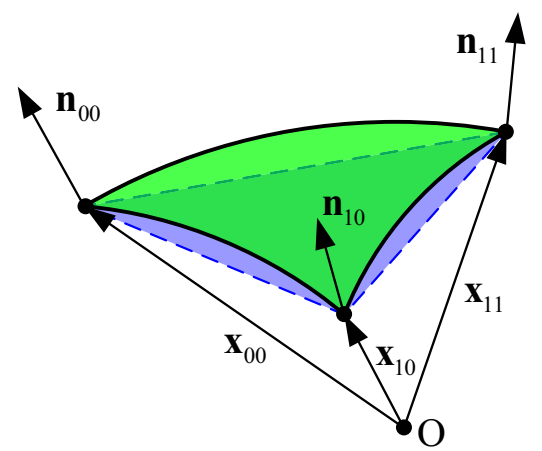

(a)

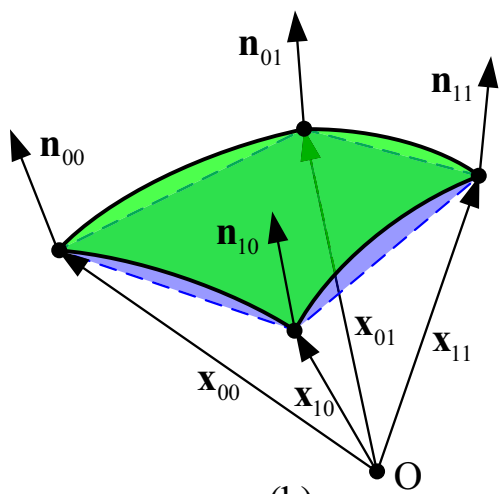

(b) 


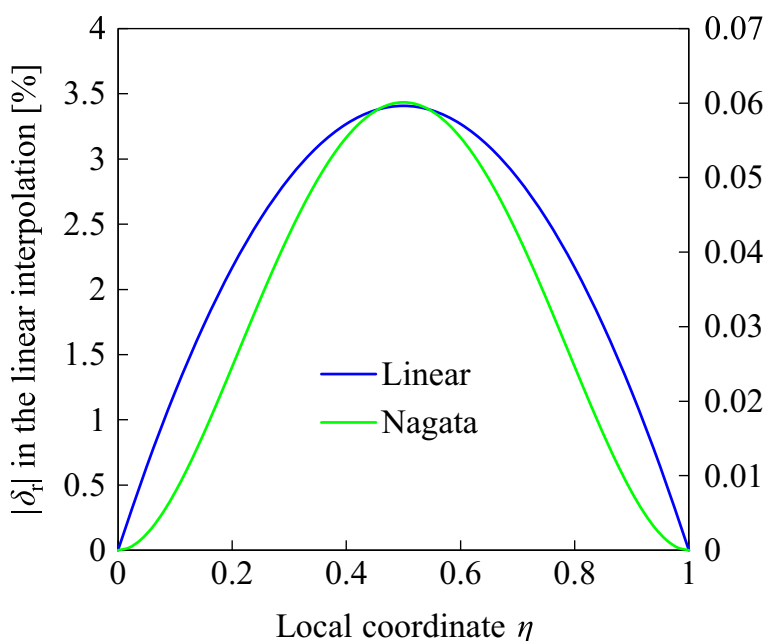

(a)

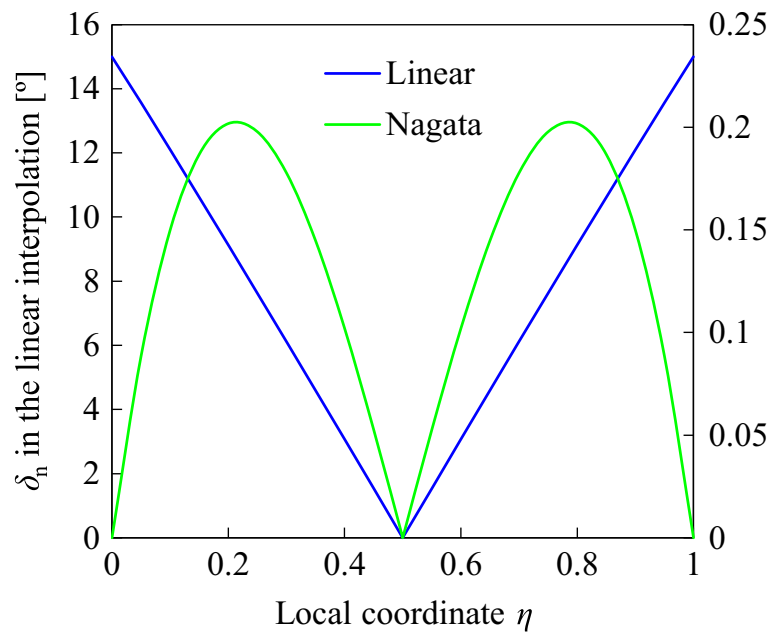

(b)

Fig. 3 Comparison between linear and Nagata interpolation applied to circular arc with $30^{\circ}$ of central angle: (a) radial error distribution; (b) normal vector error distribution

discontinuity of the normal vector in the typical finite element discretization with linear elements can be observed through the higher error at the nodes. On the other hand, the Nagata interpolation assures $G^{1}$ continuity in the interpolated curve, since the normal vector error is zero at the nodes, as shown in Fig. 3 (b). The maximum error of the normal vector in the linear interpolation is $15^{\circ}$ (always half value of the arc central angle), whereas in the Nagata interpolation is only about $0.2^{\circ}$, dictating the use of two different scales to plot the results.

The evolution of the maximum error value in function of the normalized arc length $(\ell / r)$, i.e. the discretization refinement, is presented in Fig. 4. The range considered for the normalized arc length is from 0.785 until 0.157 , which corresponds to dividing a quarter of circle from 2 to 20 equal partitions, respectively. Figure 4 (a) presents the comparison

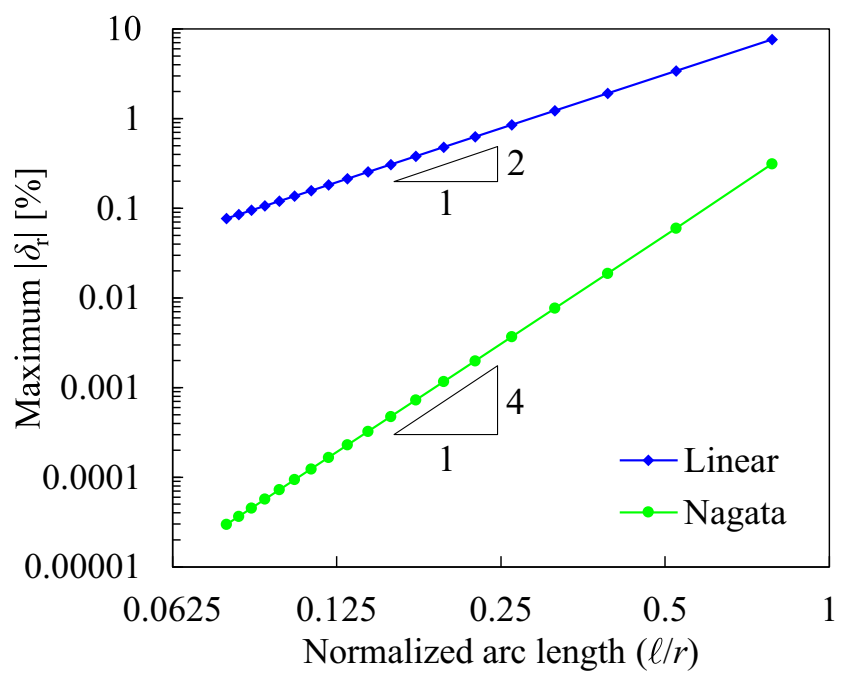

(a)

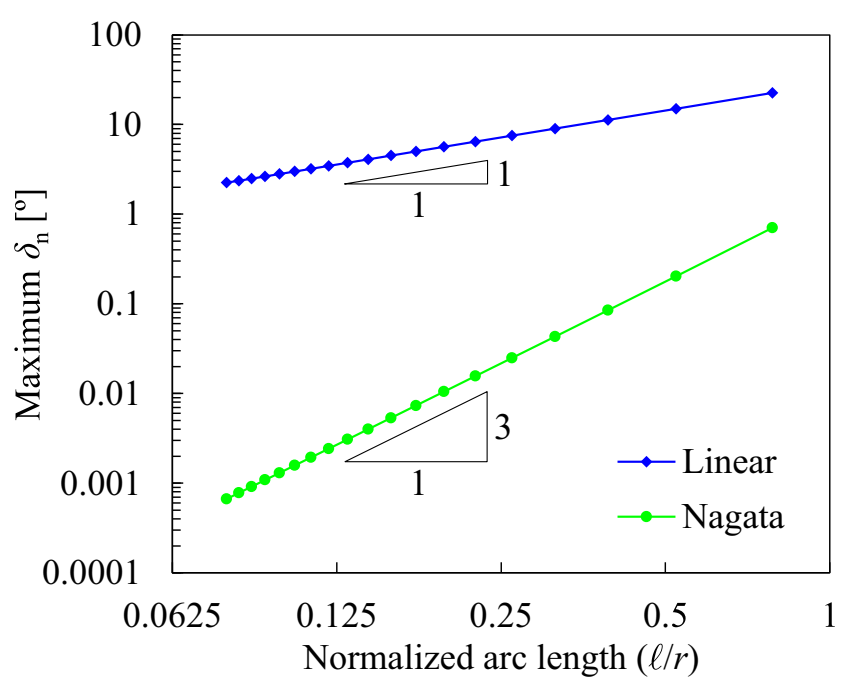

(b)

Fig. 4 Evolution of the maximum error attained in the interpolation as function of the normalized arc length: (a) maximum radial error; (b) maximum of the normal vector error

of the maximum norm of the radial error between the linear and the Nagata interpolation, as function of the normalized arc length. Considering the linear interpolation, the maximum value of the radial error decreases quadratically with the normalized arc length, while applying Nagata interpolation the convergence rate is quartic [30, 32]. Fig. 4 (b) contains the maximum normal vector error as function of the normalized arc length, for both surface description methods. The error decreases linearly when the linear interpolation is adopted, while the Nagata interpolation method provides a cubic convergence rate. These results confirm the weakness of the typically finite element discretization in terms of normal vector orientation, due to the well-known facetization induced phenomenon. 


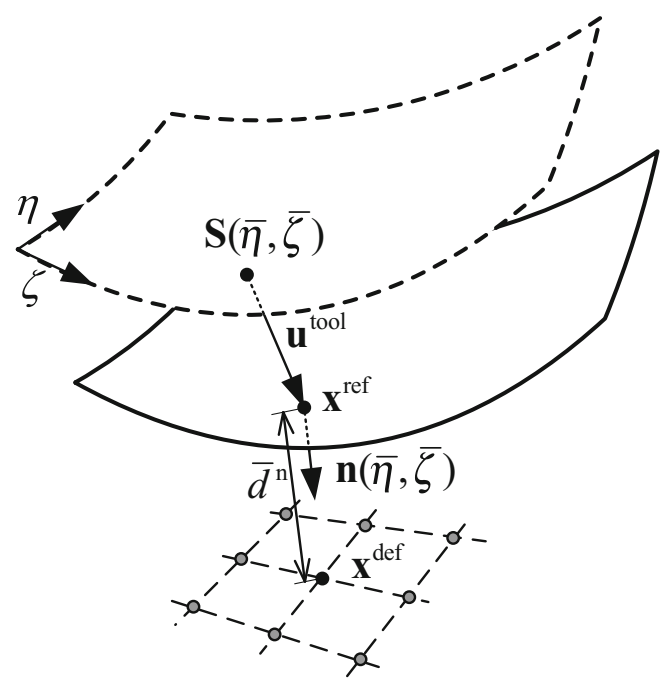

Fig. 5 Orthogonal projection of a generic node of the deformable body on a patch composing the tool surface

\section{Contact search algorithm}

The number of facets or patches required to define each tool involved in the sheet metal forming process depends on the geometric complexity of the model. The aim of the contact search algorithm is to identify, for each node of the deformable body candidate to establish contact, both the tool facet/patch and the exact position where contact can occur. The contact search algorithm must be simultaneously accurate and efficient in order to quickly identify all potential contact zones [33]. Thus, usually it is divided in two phases: global and local search. The global search algorithm must select all potential contact facets/patches, while the local contact search algorithm must identify the position where the node will establish contact. The main motivation for global contact search is to minimize the number of operations of the local search algorithm, which is usually computationally more expensive $[34,35]$.
Table 1 Elastic and plastic material properties of the HSS used in the unconstrained cylindrical bending problem

\begin{tabular}{lll}
\hline Elastic proprieties & $\begin{array}{l}\text { Swift hardening } \\
\text { law } \sigma=K\left(\varepsilon_{0}+\bar{\varepsilon}^{\mathrm{p}}\right)^{n}\end{array}$ & $\begin{array}{l}\text { Hill'48 yield } \\
\text { criterion }\end{array}$ \\
\hline$E=222 \mathrm{GPa}$ & $K=645.3 \mathrm{MPa}$ & $F=0.254$ \\
$\nu=0.30$ & $\varepsilon_{0}=0.0102$ & $G=0.397$ \\
$n=0.252$ & $H=0.603$ \\
& & $L=M=1.500$ \\
& $N=1.689$ \\
\hline
\end{tabular}

Global search

The first step of the global search algorithm involves the association of a predefined set of tools to each deformable node, based only on the orientation of each facet/patch outward normal vector to the blank. This step is carried out always at the beginning, whatever the tool surface description method adopted. The contact search algorithm implemented in DD3IMP for Bézier patches [26], which has been continuously tested and optimized, exploits the special features of Bézier patches to ensure proper and efficient contact detection. Detailed information about its particular features can be found in Oliveira et al. [26]. The main difference between both types of parametric patches lies in the number of patches needed to properly describe the tools geometry. Since the Nagata patch is only a quadratic degree surface, typically it is necessary to use more Nagata patches to attain the same accuracy as using Bézier. The same idea is also valid when the tool surfaces are described by a finite element mesh, which requires an amount of finite elements much higher to attain the same geometric accuracy, as can be observed in Fig. 4.

The global contact search algorithm for Bézier patches can be divided in the following three steps: (i) construction of a uniform grid of points on each patch; (ii) evaluation of the
Fig. 6 Scheme of the unconstrained cylindrical bending setup, including tools dimension in $\mathrm{mm}$

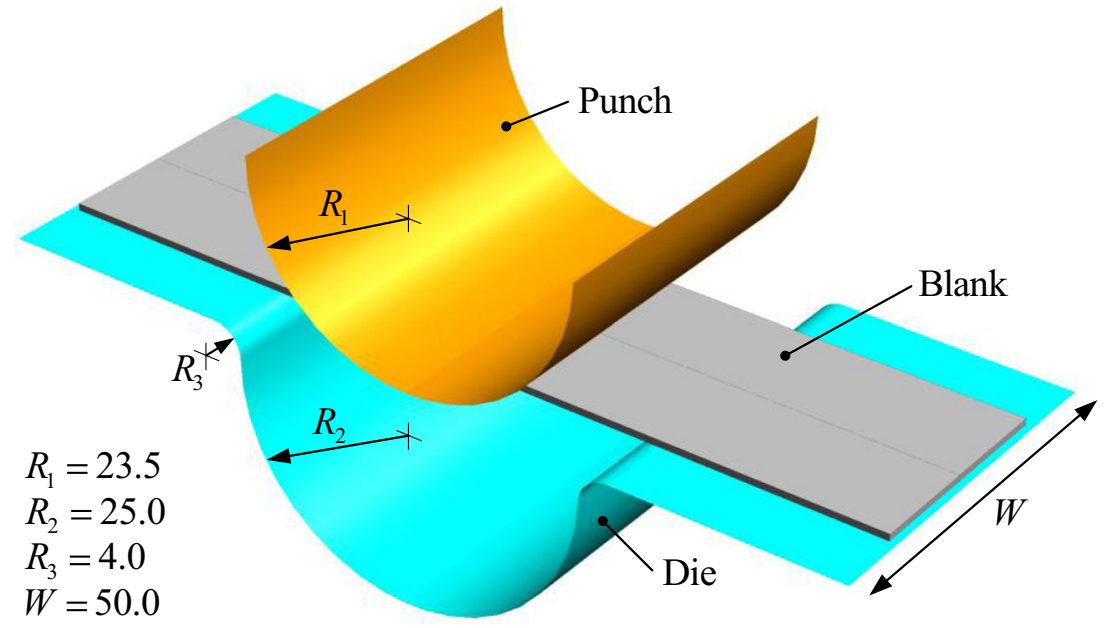


Fig. 7 Forming tool surfaces described by: (a) Bézier patches; (b) Nagata patches; (c) coarse mesh of faceted elements; (d) fine mesh of faceted elements

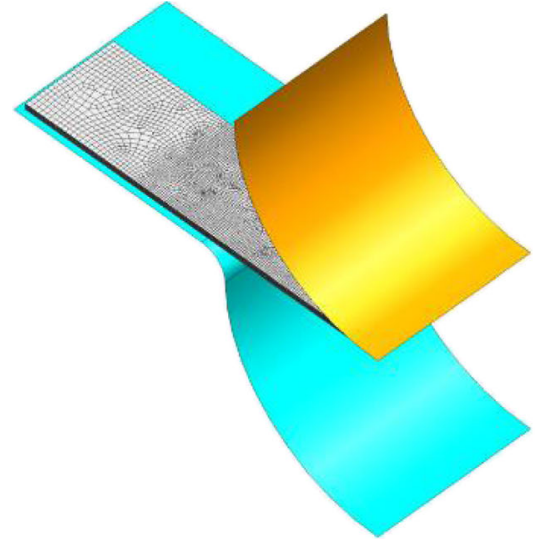

(a)

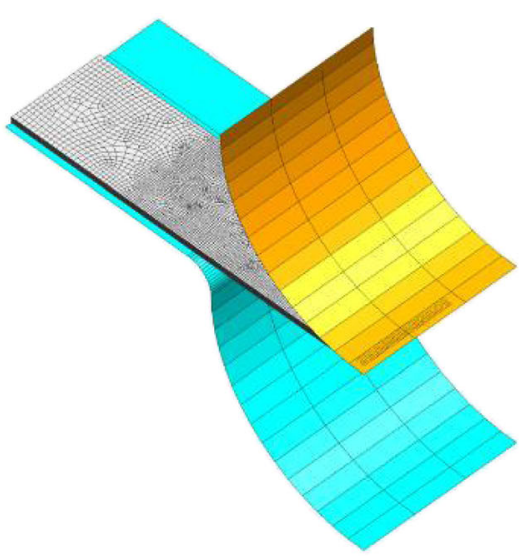

(c)

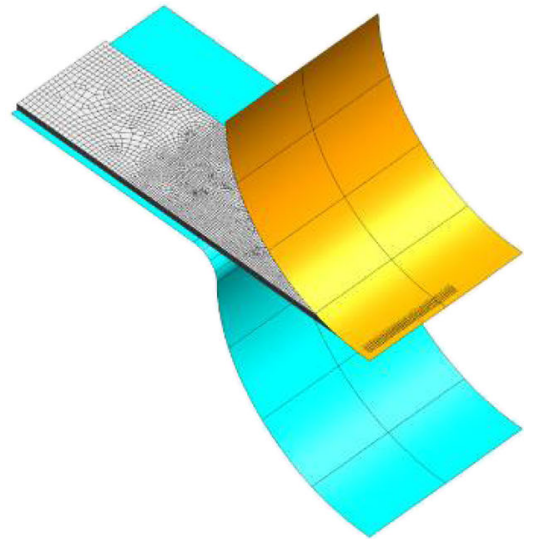

(b)

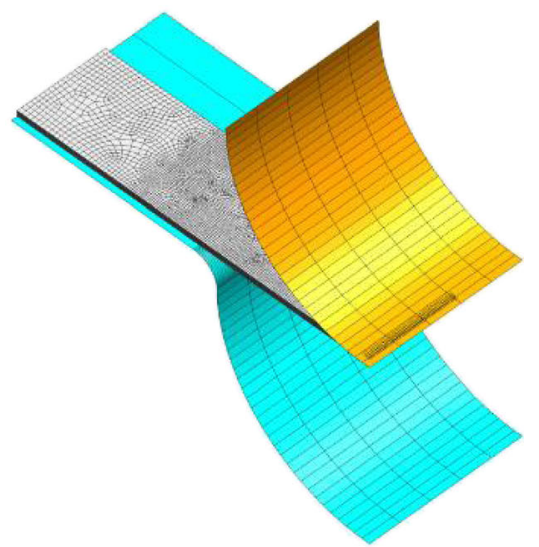

(d) distances between each node of the deformable body and the grid points, and (iii) selection of ten Bézier patches to perform the local contact search. The dimension of the grid is determined based on the maximum degree of the patch, while the

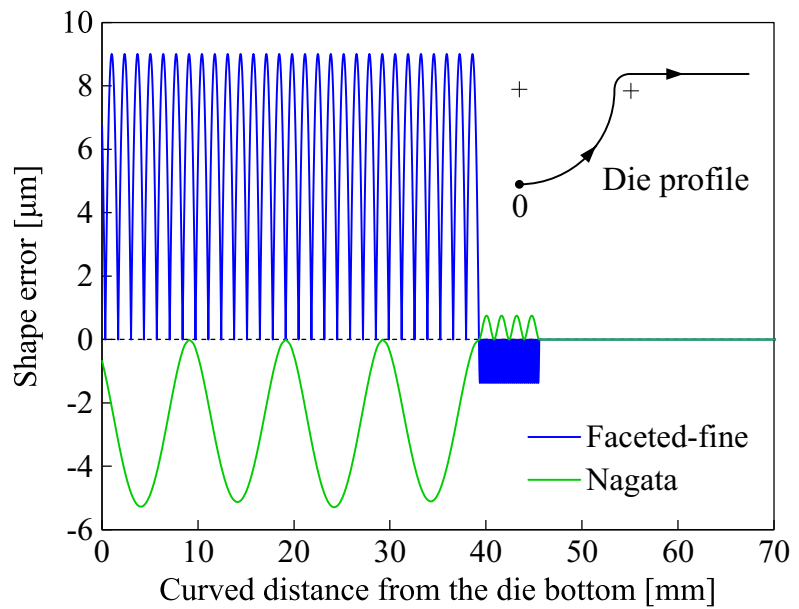

Fig. 8 Shape error distribution measured in the die profile applying both the faceted and the smoothed tool surface descriptions selection procedure in the third step is performed using the minimum distance [26].

Concerning the Nagata patch tool surface description, the global contact search algorithm was developed exploring its

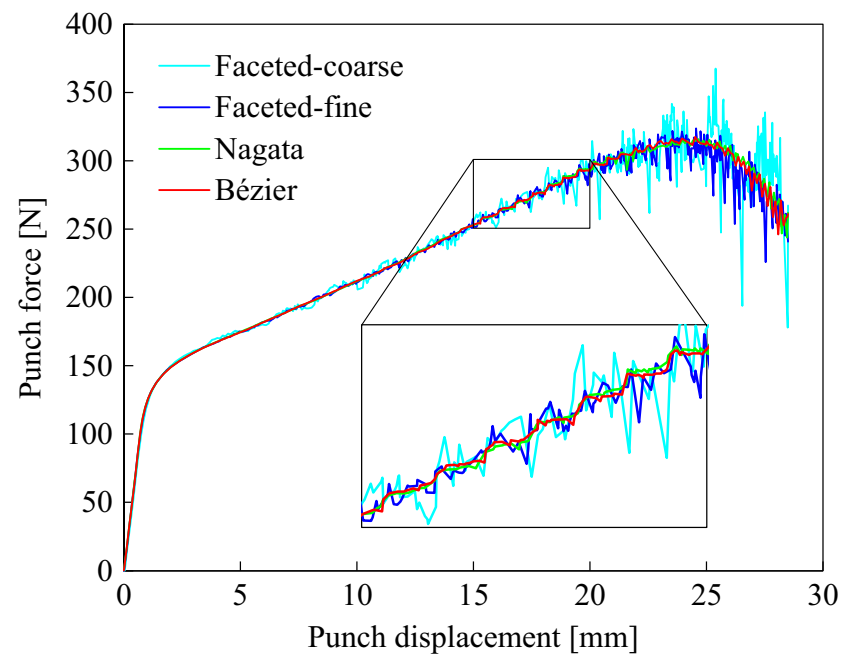

Fig. 9 Punch force evolution in the unconstrained cylindrical bending predicted with the presented tool surface description methods 
special features, such as the mesh connectivity and the coordinates of the surface mesh vertices [29]. The selection of the patches is based mainly in geometric considerations, which is divided in three steps: (i) selection of a reduced number of closest surface mesh vertices and application of inverse mesh connectivity to select their corresponding patches; (ii) creation of a uniform grid of points on each of the selected patches and (iii) selection of the ten closest Nagata patches for local contact search, based on the minimum distance between the candidate node and the grid of points. The amount of mesh vertices selected
Fig. 10 Definition of the angles between the two contact points farthest from the centreline for four different punch strokes, using the tool surfaces described by Bézier patches
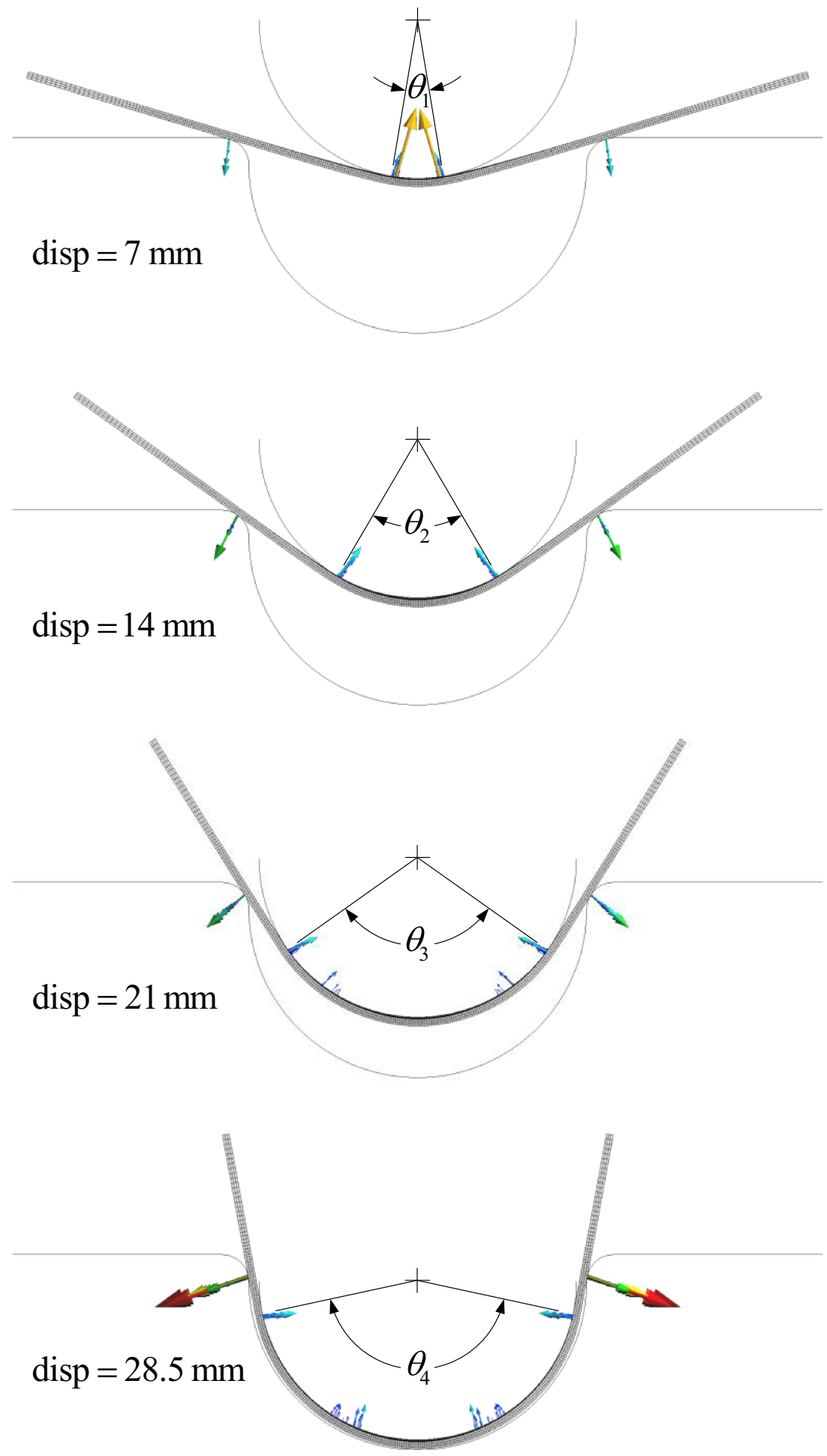
Table 2 Predicted angles between the two contact points farthest from the centreline for four different punch strokes, as well as the angle before and after springback, for the different tool surface description methods

\begin{tabular}{lllll}
\hline Angle & Bézier & Nagata & Faceted-coarse & Faceted-fine \\
\hline$\theta_{1}$ & 18.4 & 18.4 & 20.0 & 19.7 \\
$\theta_{2}$ & 60.9 & 61.7 & 56.7 & 62.9 \\
$\theta_{3}$ & 109.4 & 110.4 & 106.5 & 112.5 \\
$\theta_{4}$ & 155.3 & 155.7 & 155.9 & 155.6 \\
$\beta_{1}$ & 20.7 & 20.7 & 20.7 & 20.8 \\
$\beta_{2}$ & 35.2 & 35.0 & 33.6 & 34.9 \\
\hline
\end{tabular}

in the first step depends on the tool mesh topology, i.e. unstructured tool surface meshes require the selection of a higher number of vertices due to its irregular distribution. The dimension of the grid used in the second step is determined following a similar strategy, using the ratio between the maximum and minimum edge length of each finite element in its definition. More details about the algorithm and its numerical implementation can be found in Neto et al. [29].

\section{Local search}

In frictional contact problems involving a significant amount of sliding, the master/slave contact strategy combined with the node-to-segment discretization technique are usually adopted [36]. Therefore, the aim of the local search is to identify for each node of the deformable body (slave node) its counterpart position on the discretized tool surface (master segment). Whatever the tool surface description method adopted, the information provided by the global search is the set of the ten closest patches and the local coordinates of the closest grid point. Therefore, the orthogonal projection is applied to each patch, in order to minimize the distance between the node and the reference position [37], as shown in Fig. 5. Hence, for each node of the deformable body $\mathbf{x}^{\text {def }}$, the implicit coordinates of the
Table 3 Computational efficiency of the unconstrained cylindrical bending numerical simulation using different tool surface description methods

\begin{tabular}{lllll}
\hline & Bézier & Nagata & Faceted-coarse & Faceted-fine \\
\hline $\mathrm{N}^{\circ}$ increments & 338 & 342 & 517 & 447 \\
$\mathrm{~N}^{\circ}$ iterations & 3994 & 3918 & 4839 & 4439 \\
$\mathrm{CPU}$ time [minutes] & 94.6 & 94.5 & 112.1 & 95.1 \\
\hline
\end{tabular}

reference position $\mathbf{x}^{\text {ref }}$ and the normal distance $d^{\mathrm{n}}$ are evaluated by solving the following system of equations:

$$
\mathbf{F}^{\operatorname{Proj}}\left(\bar{\eta}, \bar{\zeta}, d^{\mathrm{n}}\right)=\mathbf{S}(\bar{\eta}, \bar{\zeta})+\mathbf{u}^{\text {tool }}+d^{\mathrm{n}} \mathbf{n}(\bar{\eta}, \bar{\zeta})-\mathbf{x}^{\mathrm{def}}=\mathbf{0},
$$

where $\mathbf{S}(\eta, \zeta)$ represents any point on the selected patch, $\mathbf{u}^{\text {tool }}$ is the tool displacement from the beginning until the actual position and $\mathbf{n}(\eta, \zeta)$ is the outward patch normal. Since the patch definition and its outward normal vector are non-linear functions of the parametric coordinates $(\eta, \zeta)$, an iterative method is mandatory to solve the problem. The Newton-Raphson algorithm is used to solve this system of equations, where the initial solution is defined by the closest grid point. The system of equations is solved for each patch preselected in the global contact search, and in case of multiple solutions, the solution with the minimum normal distance value is selected $[26,38]$.

\section{Numerical examples}

In order to quantify the effect of tool surface description method in the numerical results provided by the finite element analysis of the sheet metal forming process, two examples are studied in this section. The first one is the unconstrained cylindrical bending, proposed as benchmark of the Numisheet 2002 conference [39]. The other one is the reverse deep drawing of a cylindrical cup, proposed as benchmark at the Numisheet 1999 conference [40]. Although these two forming processes involve

Fig. 11 Deformed shape of the sheet in the unconstrained bending problem and angle definition: (a) after forming; (b) after springback

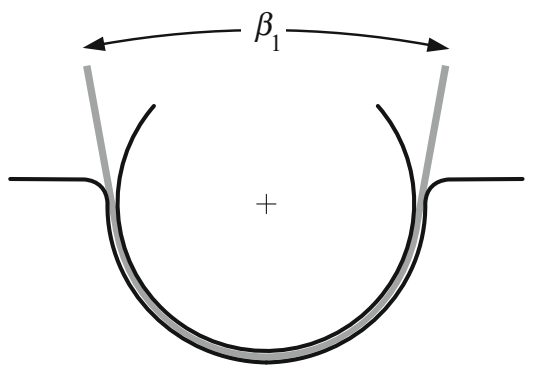

(a)

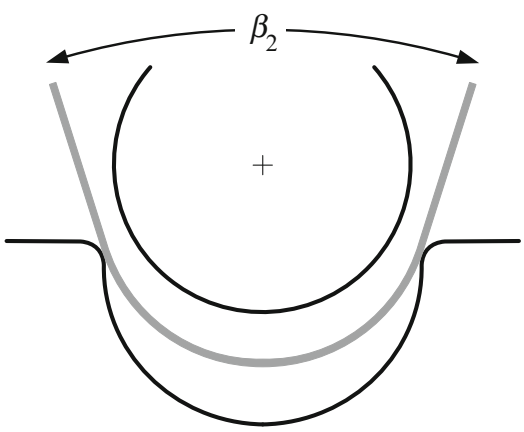

(b) 
quite simple tool geometries, the results show that they can be effectively used to compare the presented surface description methods. All simulations were carried out with DD3IMP finite element code in a computer equipped with Intel ${ }^{\circledR}$ Core $^{\mathrm{TM}}$ i72,600 K (3.4 GHz) CPU and the Windows7 Professional (64bits platform) operating system.

\section{Unconstrained cylindrical bending}

The unconstrained cylindrical bending of a high strength steel sheet, proposed as benchmark at the Numisheet 2002 conference [39] to evaluate the springback prediction, is illustrated in Fig. 6. The initial rectangular blank presents $120 \mathrm{~mm}$ of length and $30 \mathrm{~mm}$ of width, while the sheet thickness is $1 \mathrm{~mm}$. The rolling direction is aligned with the length direction and the tool dimensions are indicated in Fig. 6. The friction coefficient between the blank and the tools is taken as $\mu=$ 0.148 . The elastic and plastic properties of HSS are listed in Table 1. Due to geometric and material symmetry conditions, only one quarter of the model is simulated. The blank is discretized with an unstructured mesh composed by 19950 trilinear hexahedron solid finite elements, using three layers in the thickness direction. Since this example involves complex contact conditions due to linear contact at the die radius, unstructured blank meshes are favoured [41].

The tool surfaces are described using the three methods presented in the paper, creating two models for the faceted surface description, one with a coarse mesh and another with a fine mesh, as shown in Fig. 7. The geometrical accuracy of the tool surfaces models is evaluated through the shape error, which is defined as the distance between approximated and exact (CAD model) definition of the surfaces. Figure 8 presents the comparison of the shape error distribution obtained with the fine mesh of facets and the Nagata smoothing method. Although the tool model composed by facets uses 30 finite elements to describe each arc of circle section (see Fig. 7 (d)), in opposition to only 4 Nagata patches in the smoothing method (see Fig. 7 (b)), the maximum shape error value $(9 \mu \mathrm{m})$ is reached by the faceted tool surface description. Moreover, the error in the normal vector direction present in the faceted tool model is approximately $1.5^{\circ}$, while in the Nagata smoothing method it is inferior to $0.1^{\circ}$, as shown in Fig. 4 (b). The comparison of the punch force evolution obtained with the different tool surface description methods is shown in Fig. 9. Concerning the faceted tool surface description, the mesh refinement (i.e. increase of the number of facets used) reduces the oscillations in the punch force evolution. Nevertheless, only the smoothing method with Nagata patches and the tool definition using Bézier patches leads to a force evolution with irrelevant oscillations, as can be observed in the zoom view of Fig. 9. The angles between two contact points that are the farthest from the centreline at 7, 14, 21 and $28.5 \mathrm{~mm}$ of punch stroke are illustrated in Fig. 10, for the tool

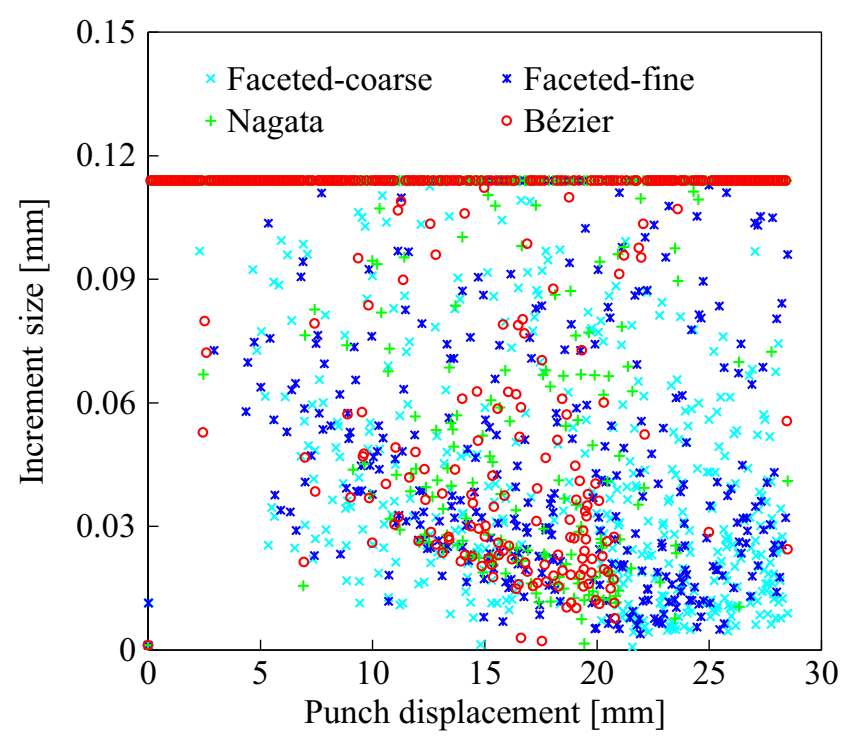

Fig. 12 Increment size evolution with the punch displacement for the unconstrained cylindrical bending problem

surfaces described by Bézier patches. The predicted values of these angles are presented in Table 2 for all tool surface description methods analysed. Only the faceted tool surface description with the coarse mesh (see Fig. 7 (c)) gives dissimilar results to the ones obtained with the other models. Nevertheless, the angles predicted with the tool surfaces described by Nagata patches are closer to the one obtained when applying Bézier patches. The amount of springback is evaluated through the angles measured after forming at the maximum punch displacement and after springback, which are defined in Fig. 11. Table 2 contains the predicted angles values for the four tool models considered in this study. While the angle after forming is rather insensitive to the tool surface description method, after springback the use of the coarse mesh in the faceted model provides a lower springback angle. Indeed, after forming the sheet is enclosed between the tools, which dictate its geometry. On the other hand, after springback the stress field produced during the forming process is replicated in the final

Table 4 Elastic and plastic material properties of the DDQ steel used in the reverse deep drawing problem

\begin{tabular}{lll}
\hline $\begin{array}{l}\text { Elastic } \\
\text { proprieties }\end{array}$ & $\begin{array}{l}\text { Swift hardening } \\
\text { law } \sigma=K\left(\varepsilon_{0}+\bar{\varepsilon}^{\mathrm{p}}\right)^{n}\end{array}$ & $\begin{array}{l}\text { Hill'48 yield } \\
\text { criterion }\end{array}$ \\
\hline$E=210 \mathrm{GPa}$ & $K=568.3 \mathrm{MPa}$ & $F=0.3137$ \\
$\nu=0.30$ & $\varepsilon_{0}=0.0059$ & $G=0.3663$ \\
& $n=0.233$ & $H=0.6337$ \\
& & $L=M=1.500$ \\
& $N=1.1764$ \\
\hline
\end{tabular}


shape, where the accuracy of the tool surface description is an important variable [13].

Concerning the computational efficiency, the total number of increments and equilibrium iterations as well as the CPU time are compared in Table 3. The number of increments decreases with the mesh refinement in the faceted tool surface description, which is always lower for the Nagata and Bézier description methods. The same trend is observed for the total number of equilibrium iterations. Regarding the CPU time, only the coarse mesh composed by facets requires more computational time, which is about $20 \%$ higher than the one required by the other models. Figure 12 presents the increment size evolution with the punch displacement. Since an $r_{\min }$ strategy $[41,42]$ is implemented to automatically limit the increment size in order to improve convergence, the real increment size can be inferior to the one defined in input (in this case $0.114 \mathrm{~mm}$ ). This automatic reduction is more evident for the faceted tool surface description method, mainly for a punch displacement higher than $20 \mathrm{~mm}$. In fact, by adopting a smoothed tool surface description (Nagata or Bézier), most of the increments present the size imposed as input (see Fig. 12). Note that the difference between Nagata and Bézier tool
Table 5 Number of patches/facets used to describe each forming tool of the reverse deep drawing problem

\begin{tabular}{lllll}
\hline Tool & Bézier & Nagata & Faceted-coarse & Faceted-fine \\
\hline Die 1 & 8 & 90 & 324 & 960 \\
Blank-holder 1 & 2 & 15 & 60 & 180 \\
Punch 1/Die 2 & 12 & 120 & 540 & 1680 \\
Blank-holder 2 & 8 & 75 & 240 & 840 \\
Punch 2 & 12 & 86 & 254 & 1012 \\
Total & 42 & 386 & 1418 & 4672 \\
\hline
\end{tabular}

surface description methods is very small for all parameters analysed, while the construction of the tool model with Nagata patches is simplest and easier.

\section{Reverse deep drawing of a cylindrical cup}

The second example analysed is the reverse deep drawing process of a cylindrical cup, proposed as benchmark at the Numisheet'99 conference [40]. The circular blank has
Fig. 13 Forming tools adopted in the reverse deep drawing using the surfaces described by: (a) Bézier patches; (b) Nagata patches; (c) coarse mesh of faceted elements; (d) fine mesh of faceted elements

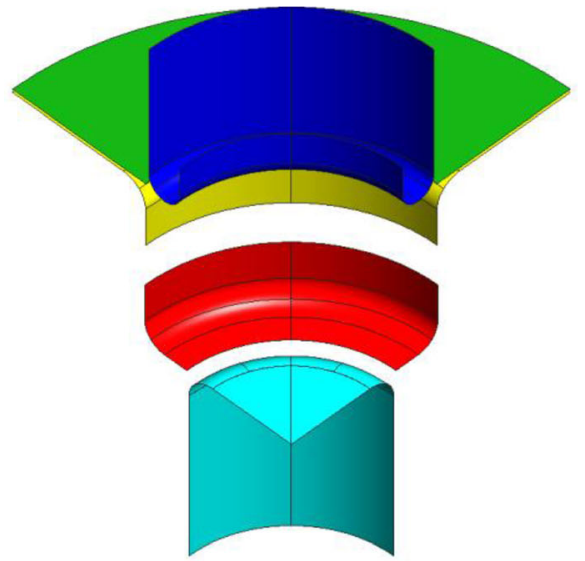

(a)

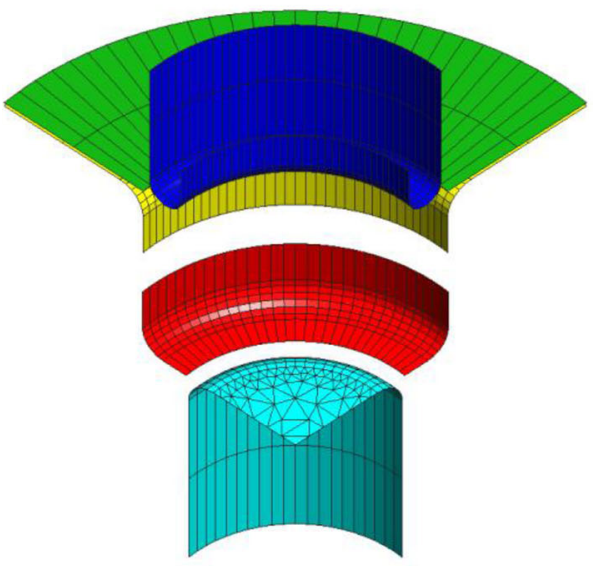

(c)

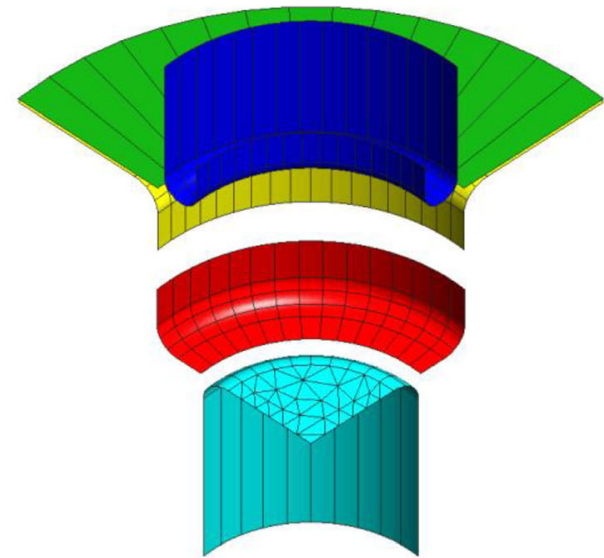

(b)

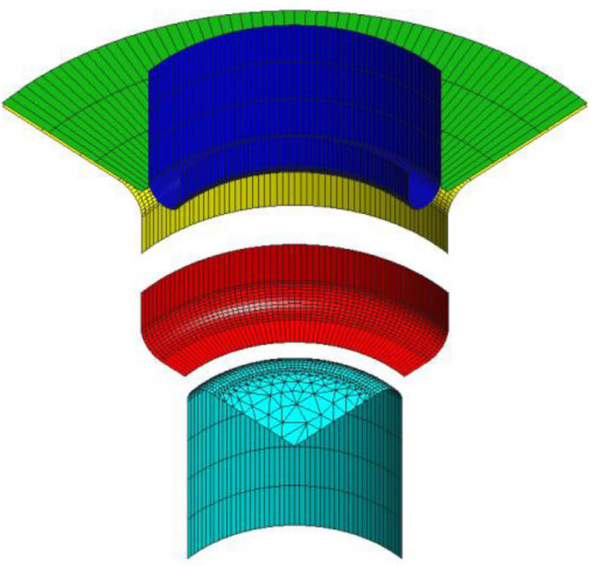

(d) 
$170 \mathrm{~mm}$ diameter and $0.98 \mathrm{~mm}$ of initial thickness. The material considered in this study is the mild steel DDQ, characterized by the elastic and plastic properties listed in Table 4. The friction coefficient between the sheet and the tools is taken from the benchmark specifications as $\mu=0.15$. Due to geometric and material symmetry conditions, only one quarter of the model is simulated, as shown in Fig. 13. The blank is discretized with 8-node hexahedron solid finite elements, using 2 layers through the thickness, making a total of 15408 elements. The gap between the die and the blank-holder is held fixed in both stages, which is set equal to $1.13 \mathrm{~mm}$ in the first stage and $1.4 \mathrm{~mm}$ in the second stage, in order to draw a cylindrical cup without wrinkles [43, 44].

The three methods presented in the paper are used to describe the tool surfaces, as shown in Fig. 13. Two models for the faceted surface description (coarse and fine mesh) are created. The model composed by Nagata patches uses 3 patches to describe each circular arc in the radial direction (see Fig. 13 (b)), while in the faceted surface description method 6 and 12 bilinear facets are applied for the coarse and fine mesh (see Fig. 13 (c) and (d)), respectively. The total number of patches or facets composing each tool model is

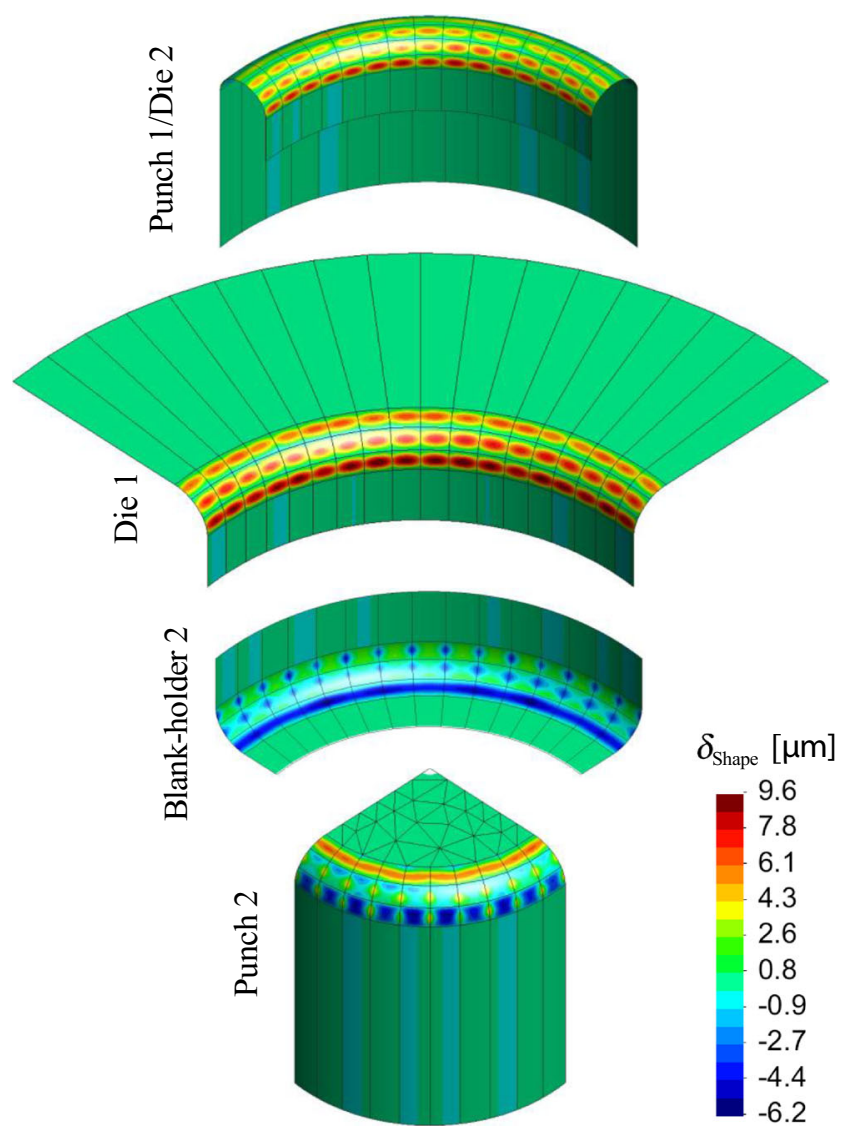

Fig. 14 Shape error distribution on the tool surfaces described by Nagata patches presented in Table 5. The shape error is selected to assess the geometrical accuracy of the tool surfaces models, comparing all surface description methods that are based on the discretization of the tool surfaces. Figure 14 presents the shape error distribution on the tool surfaces described by Nagata patches. The maximum positive error appears in the hyperbolic surface of the die, while the maximum negative error occurs in elliptic surface of the punch 2. Nevertheless, the shape error ranges from -6.2 to $9.6 \mu \mathrm{m}$, thus the maximum value attained always inferior to $10 \mu \mathrm{m}$. The comparison of the shape error distribution obtained with the faceted tool surface description for the coarse and fine mesh is presented in Fig. 15 and Fig. 16, respectively. Although the finer tool model composed by facets uses much more facets than Nagata patches to describe the contact surfaces (see Table 5), the maximum error value reached is approximately twice than using Nagata patches. Moreover, the sudden variation of the shape error within each facet, which can be observed in Fig. 15 and Fig. 16, combined with small size facets leads to a higher error in the normal vector direction.

The comparison of the predicted punch force evolution using different tool surface description methods is presented in Fig. 17 and Fig. 18, for the first and second forming stage,

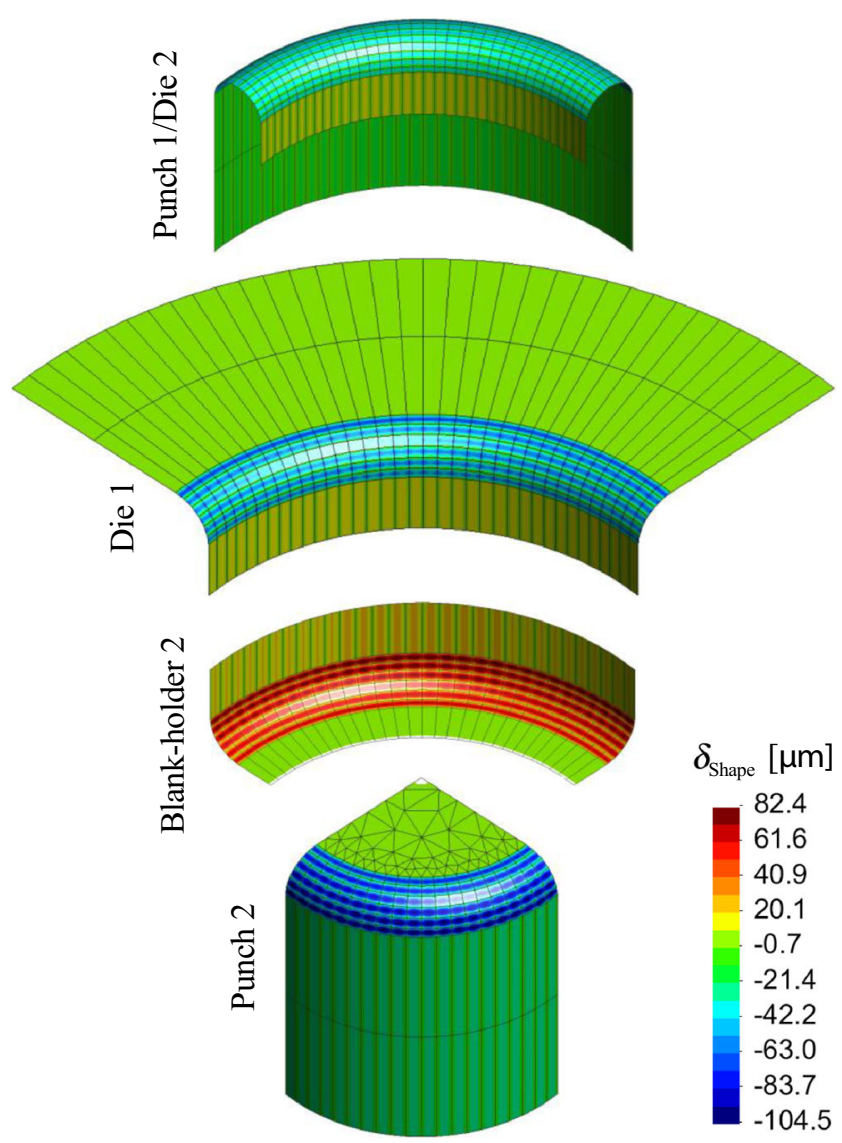

Fig. 15 Shape error distribution on the tool surfaces described by bilinear facets, adopting a coarse mesh 


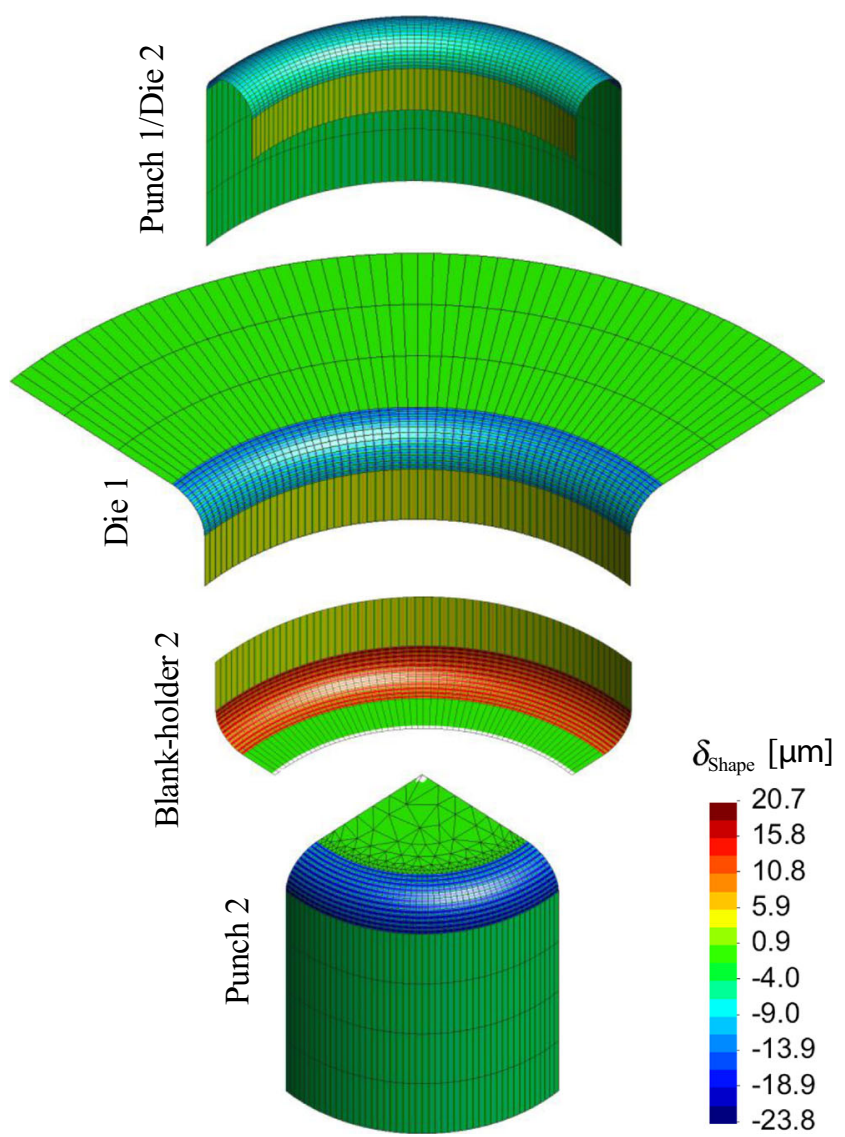

Fig. 16 Shape error distribution on the tool surfaces described by bilinear facets, adopting a fine mesh

respectively. The effect of the tool surface accuracy can be observed in both forming stages. Nevertheless, it is more emphasised during the second stage due to the lower value of the die radius. Regarding the faceted tool surface

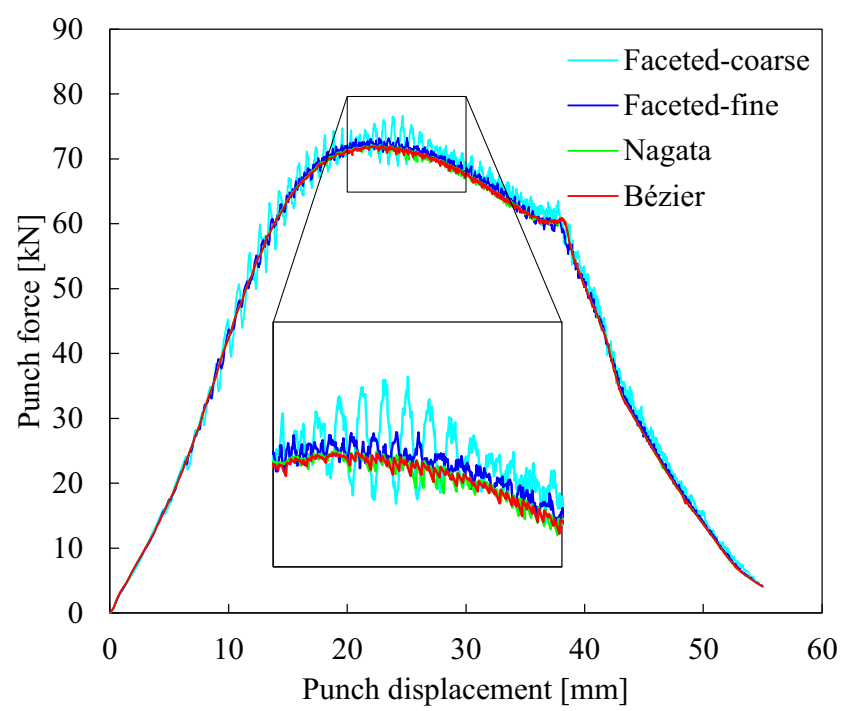

Fig. 17 Punch force evolution during the 1st stage, obtained with different tool surface description methods

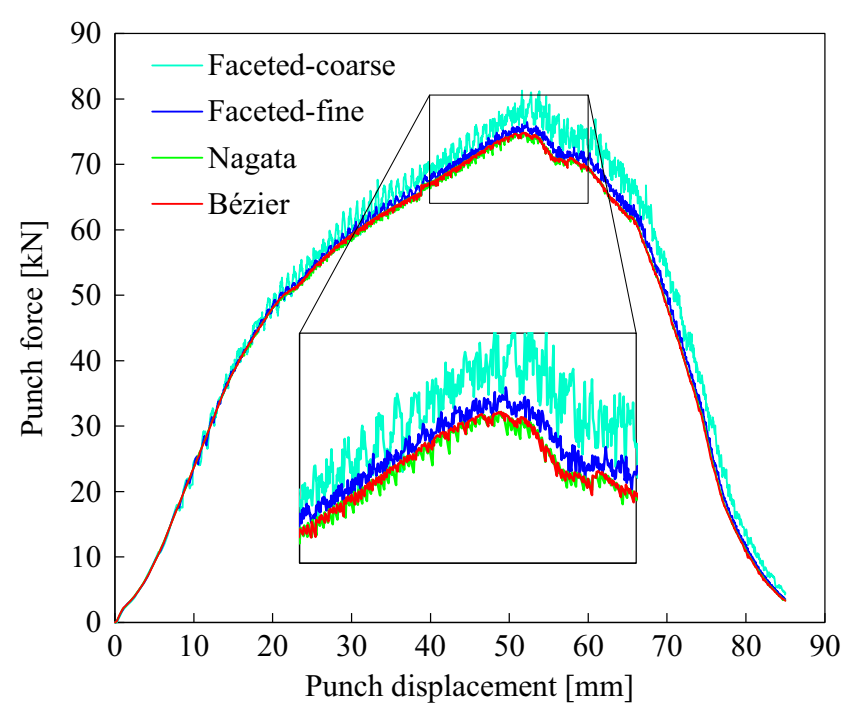

Fig. 18 Punch force evolution during the 2nd stage, obtained with different tool surface description methods

description, the increase of the number of facets reduces the oscillations in the punch force evolution. However, only the surface smoothing method with Nagata patches and the tools defined by Bézier patches lead to a force evolution with reduced oscillations, as can be observed in the zoom view of Fig. 17 and Fig. 18. The equivalent plastic strain distribution at the end of the second stage is presented in Fig. 19, for each tool surface description method. Both the Bézier and Nagata patches applied in the tool surface description lead to identical results, presenting in Fig. 19 (c) only the distribution obtained with Nagata patches. Indeed, the equivalent plastic strain predicted using the tool surfaces defined by facets is considerable different from the one obtained with smooth patches, particularly when adopting the coarser mesh. The evolution of the number of nodes in contact with the die, for each forming stage, is presented in Fig. 20 for each tool surface description method. The higher number of nodes in contact predicted by the tools modelled by Bézier patches is associated with the excellent geometrical accuracy in the tool surfaces definition. On the other hand, the faceted description provides a lower number of nodes in contact, particularly in the first stage (see Fig. 20 (a)), due to the artificial roughness induced in the contact surface. In fact, the mesh refinement performed in the tool surface description is more effective in the second stage than in the first one, as can be seen in Fig. 20. This is a consequence of the lower value of the die radius in the second stage and the absence of the planar surface (compare the shape error distributions for Die 1 and Punch 1/Die 2, in Fig. 15 and Fig. 16).

The computational efficiency of the numerical simulations is evaluated in this study through the number of increments, total number of equilibrium iterations and the CPU time, which are compared in Table 6. The required number of increments 
Fig. 19 Equivalent plastic strain distribution at the end of 2 nd stage, using tool surfaces described by: (a) faceted coarse mesh; (b) faceted fine mesh; (c) Nagata patches

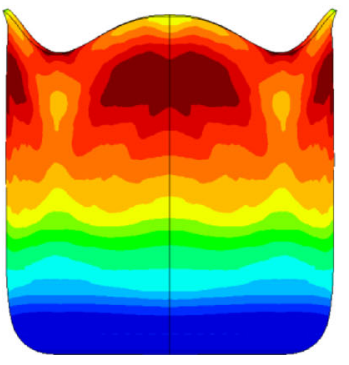

(a)

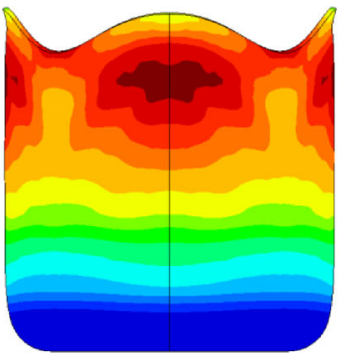

(b)

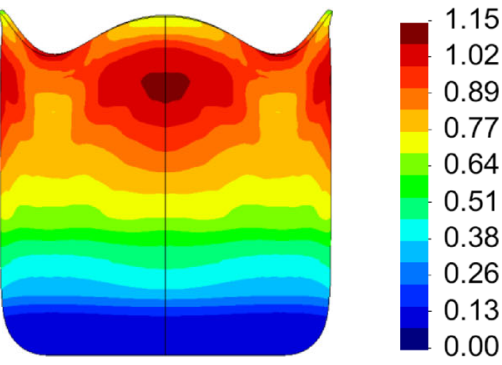

(c)

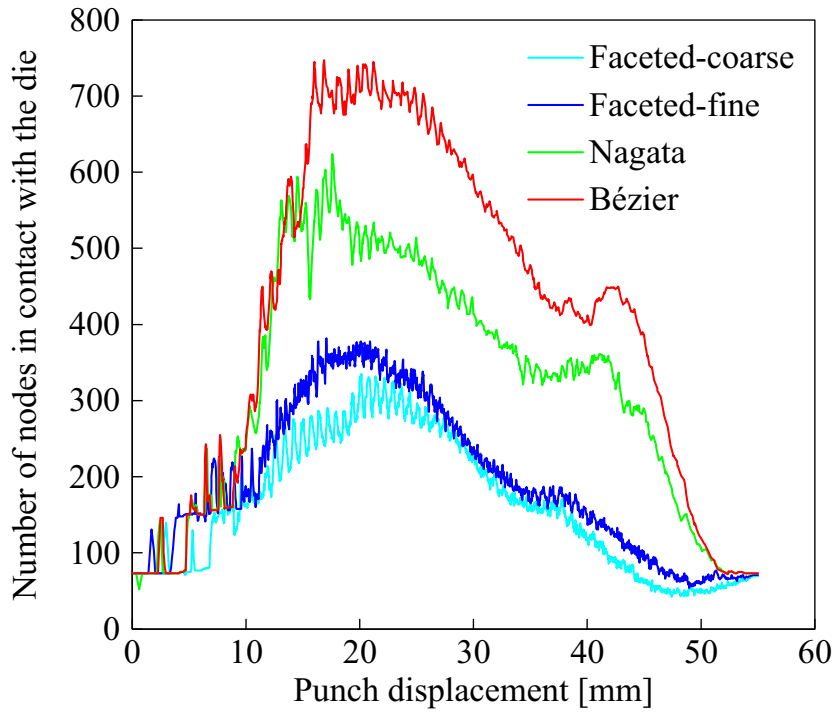

(a)

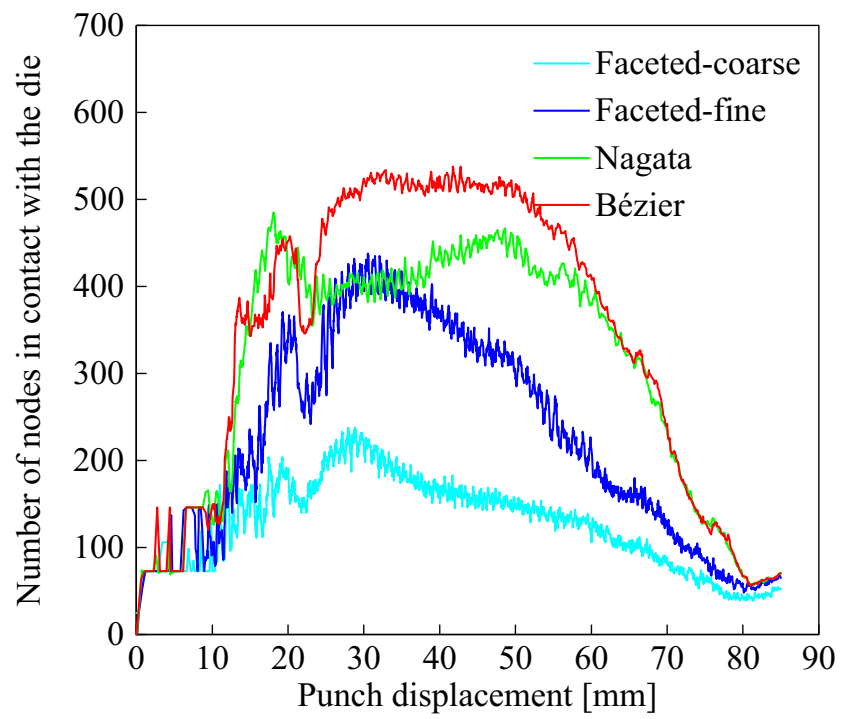

(b)

Fig. 20 Evolution of the number of nodes in contact with the die during the: (a) 1st stage; (b) 2nd stage decreases slightly with the mesh refinement in the faceted tool surface description. This is directly related with the error in the normal vector of the discretized tool surfaces, which decreases only linearly with the mesh refinement, as shown Fig. 4 (b). On the other hand, the smooth tool surface descriptions (Bézier and Nagata) require approximately half the number of increments to complete the simulation. Identical behaviour is observed for the total number of equilibrium iterations. Figure 21 presents the increment size evolution with the punch displacement for both forming stages. The reduction in the increment size defined by the $r_{\text {min }}$ strategy is more emphasised for the faceted tool surface description method (coarse and fine mesh) due to the sudden changes in the surface normal vector direction, when the sheet slides over the tool surfaces. Concerning the Bézier and the Nagata patch surface description methods, its increment size evolution is analogous, as shown in Fig. 21. The total CPU time is mainly dictated by the number of increments required to perform the simulation. Therefore, the computational time required to carry out the numerical simulation using the faceted tool surface description is approximately twice of the one required when a smooth surface description is adopted, as shown in Table 6. Although the total number of Nagata patches used in tool surface description is higher than the number of Bézier patches (see Table 5), the required CPU time to complete the simulation is slightly lower when applying Nagata patches. The same trend is observed for the total number iterations and increments. This behaviour can be associated with the high interpolation degree used in some Bézier patches, which can create instabilities in the local search.

Table 6 Computational efficiency of the reverse deep drawing numerical simulation using different tool surface description methods

\begin{tabular}{lllll}
\hline & Bézier & Nagata & Faceted-coarse & Faceted-fine \\
\hline $\mathrm{N}^{\circ}$ increments & 1917 & 1788 & 4248 & 4103 \\
$\mathrm{~N}^{\circ}$ iterations & 12925 & 12460 & 25939 & 24103 \\
CPU time [hours] & 3.62 & 3.28 & 6.41 & 6.01 \\
\hline
\end{tabular}




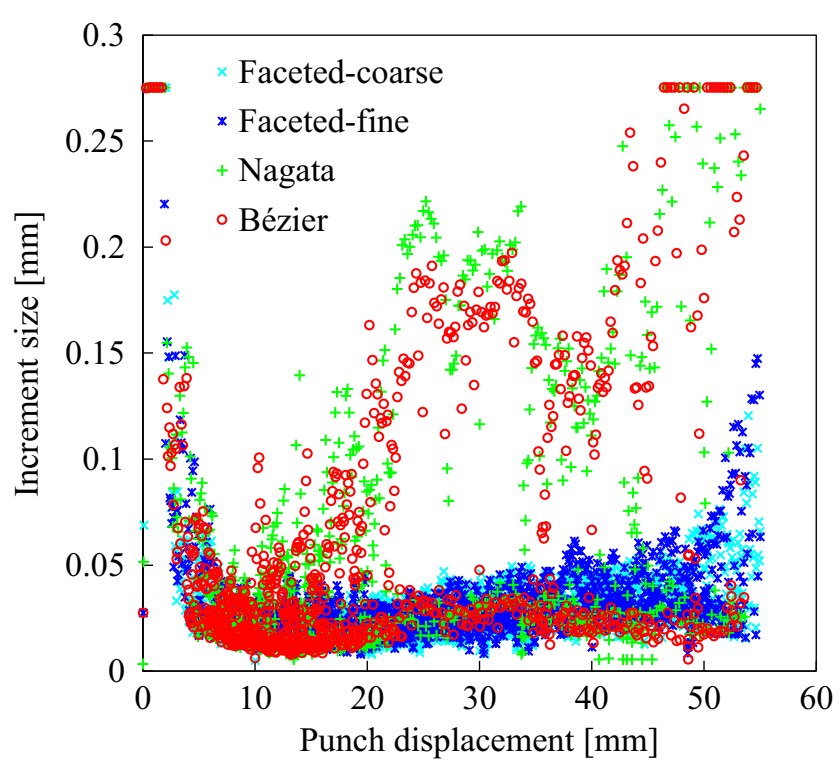

(a)

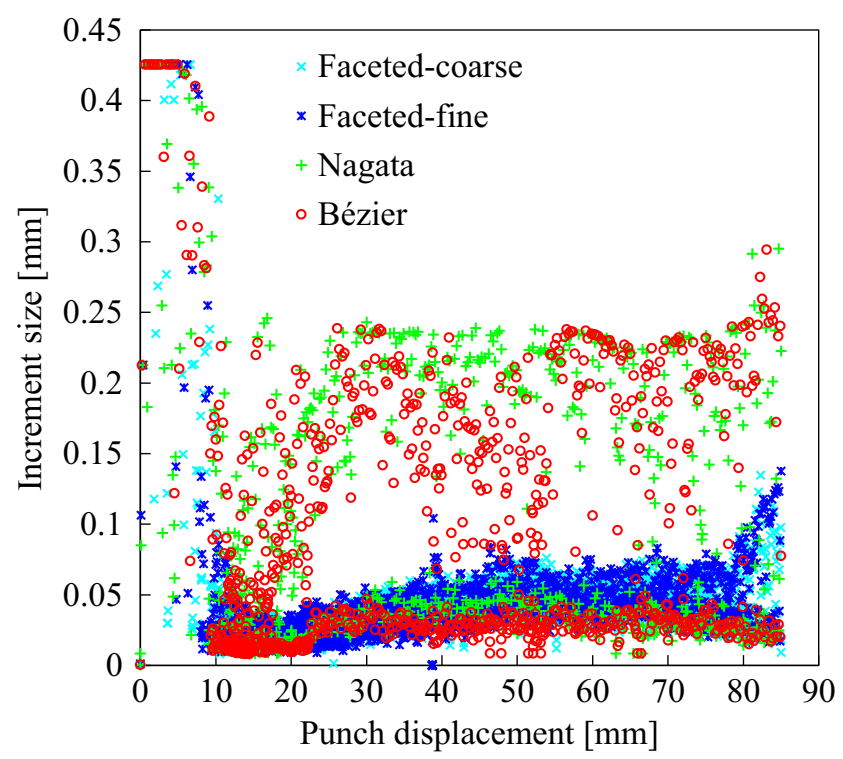

(b)

Fig. 21 Increment size evolution with the punch displacement for reverse deep drawing: (a) 1st stage; (b) 2nd stage

\section{Conclusions}

This paper presents the comparison of three different tool surface description methods used in the numerical simulation of sheet metal forming processes. The contact surfaces involved in the finite element analysis are defined using: (i) Bézier patches obtained from the CAD model, (ii) Nagata patches as result of surface mesh smoothing and (iii) bilinear facets, inherent to the use of the traditional finite element surface discretization. The contact search algorithm, which is divided in two phases: global and local search, is briefly presented for each tool surface description method. The influence of the tool model accuracy on the numerical analysis is established through two examples: the unconstrained cylindrical bending and the reverse deep drawing of a cylindrical cup.

The surface description method that resorts to Nagata patches leads to significant improvements in terms of geometric accuracy of the tool surfaces, in comparison with bilinear finite element meshes. Moreover, the surface normal vector is quasi-continuous when applying Nagata patches, which is discontinuous in the faceted surface description. Therefore, the simulation results are largely affected by the surface discretization when bilinear facets are used, creating unphysical oscillations in the punch force evolution. Besides, the predicted equivalent plastic strain distribution is overestimated due to the artificial roughness created. On the other hand, the use of Nagata patches allows obtaining an accurate tool definition without drastically increasing the number of patches. Concerning the computational efficiency, the smooth description of the tool surfaces is much more advantageous than the facets, particularly due to the normal vector accuracy. Indeed, for the example involving large slide of the sheet over the tools, both the total number of increments and the CPU time required to complete the numerical simulation when using the faceted tool surface description is approximately twice of the one required by the models using a smooth surface description. These results show that the description of the tool surfaces using Nagata patches provides accurate results, while requiring smaller computational time. Moreover, the construction of Nagata patch tool models is simplest and easier than the one based in Bézier patches, which is dependent of the CAD software.

Acknowledgements The authors gratefully acknowledge the financial support of the Portuguese Foundation for Science and Technology (FCT) via the projects PTDC/EME-TME/118420/2010 and PEst-C/EME/ UI0285/2013 and by FEDER funds through the program COMPETE Programa Operacional Factores de Competitividade, under the project CENTRO-07-0224-FEDER-002001 (MT4MOBI). The first author is also grateful to the FCT for the $\mathrm{PhD}$ grant $\mathrm{SFRH} / \mathrm{BD} / 69140 / 2010$.

\section{References}

1. Tekkaya AE (2000) State-of-the-art of simulation of sheet metal forming. J Mater Process Technol 103:14-22. doi:10.1016/S09240136(00)00413-1

2. Makinouchi A (1996) Sheet metal forming simulation in industry. J Mater Process Technol 60:19-26. doi:10.1016/0924-0136(96) 02303-5

3. Gantar G, Pepelnjak T, Kuzman K (2002) Optimization of sheet metal forming processes by the use of numerical simulations. $\mathrm{J}$ Mater Process Technol 130-131:54-59. doi:10.1016/S09240136(02)00786-0

4. Chenot J-L, Fourment L, Mocellin K (2002) Numerical treatment of contact and friction in FE simulation of forming processes. J Mater 
Process Technol 125-126:45-52. doi:10.1016/S0924-0136(02) 00416-8

5. Yoshida F, Uemori T (2002) A model of large-strain cyclic plasticity describing the Bauschinger effect and workhardening stagnation. Int J Plast 18:661-686. doi:10.1016/S0749-6419(01)00050-X

6. de Sousa RJ A, Cardoso RPR, Valente RAF, Yoon J-W, Grácio JJ, Natal Jorge RM (2005) A new one-point quadrature enhanced assumed strain (EAS) solid-shell element with multiple integration points along thickness: Part I-geometrically linear applications. Int J Numer Methods Eng 62:952-977. doi:10.1002/nme.1226

7. Hughes TJR, Cottrell JA, Bazilevs Y (2005) Isogeometric analysis: $\mathrm{CAD}$, finite elements, NURBS, exact geometry and mesh refinement. Comput Methods Appl Mech Eng 194:4135-4195. doi:10. 1016/j.cma.2004.10.008

8. Cazacu O, Barlat F (2001) Generalization of Drucker's yield criterion to orthotropy. Math Mech Solids 6:613-630. doi:10.1177/ 108128650100600603

9. Barlat F, Brem JC, Yoon JW, Chung K, Dick RE, Lege DJ, Pourboghrat F, Choi S-H, Chu E (2003) Plane stress yield function for aluminum alloy sheets-part 1: theory. Int J Plast 19:1297-1319. doi:10.1016/S0749-6419(02)00019-0

10. Menezes LF, Teodosiu C (2000) Three-dimensional numerical simulation of the deep-drawing process using solid finite elements. J Mater Process Technol 97:100-106. doi:10.1016/S0924-0136(99)00345-3

11. Yang DY, Yoo DJ, Song IS, Lee JH (1995) Investigation into tool surface description for finite element analysis of three-dimensional sheet metal forming process. J Mater Process Technol 45:267-273. http://dx.doi.org/10.1016/0924-0136(94)90351-4

12. Santos A, Makinouchi A (1995) Contact Strategies to deal with different tool descriptions in static explicit FEM of 3-D sheet metal forming simulation, J Mater Process Technol 50:277-291. http://dx. doi.org/10.1016/0924-0136(94)01391-D

13. Hama T, Nagata T, Teodosiu C, Makinouchi A, Takuda H (2008) Finite-element simulation of springback in sheet metal forming using local interpolation for tool surfaces. Int J Mech Sci 50:175-192. http://dx.doi.org/10.1016/j.ijmecsci.2007.07.005

14. Hachani M, Fourment L (2013) A smoothing procedure based on quasi- $\mathrm{C}^{1}$ interpolation for $3 \mathrm{D}$ contact mechanics with applications to metal forming. Comput Struct 128:1-13. doi:10.1016/j.compstruc. 2013.05.008

15. Shim HB, Suh EK (2000) Contact treatment algorithm for the trimmed NURBS surface. J Mater Process Technol 104:200-206. http://dx.doi.org/10.1016/S0924-0136(00)00555-0

16. Zhu X-F, Hu P, Ma Z-D, Zhang X, Li W, Bao J, Liu M (2013) A new surface parameterization method based on one-step inverse forming for isogeometric analysis-suited geometry. Int J Adv Manuf Technol 65:1215-1227. http://dx.doi.org/10.1007/s00170-012-4251-8.

17. Stadler M, Holzapfel GA, Korelc J (2003) $C^{\mathrm{n}}$ continuous modelling of smooth contact surfaces using NURBS and application to 2D problems. Int J Numer Methods Eng 57:2177-2203. http://dx.doi. org/10.1002/nme.776.

18. Krstulovic-Opara L, Wriggers P, Korelc J (2002) A $C^{1}$-continuous formulation for $3 \mathrm{D}$ finite deformation friction contact. Comput Mech 29:27-42. http://dx.doi.org/10.1007/s00466-002-0317-z.

19. Puso MA, Laursen TA (2002) A 3D contact smoothing method using Gregory patches. Int J Numer Methods Eng 54:1161-1194. http:// dx.doi.org/10.1002/nme.466.

20. McDevitt TW, Laursen TA (2000) A mortar-finite element formulation for frictional contact problems. Int J Numer Methods Eng 48: 1525-1547. http://dx.doi.org/10.1002/1097-0207(20000810) 48:10<1525::AID-NME953>3.0.CO;2-Y doi:10.1002/10970207(20000810)48:10\%3c1525::AID-NME953\%3e3.0.CO;2-Y .

21. Puso MA, Laursen TA (2004) A mortar segment-to-segment contact method for large deformation solid mechanics. Comput Method Appl Mech Eng 193:601-629. http://dx.doi.org/10.1016/j. cma.2003.10.010.
22. Puso MA, Laursen TA (2004) A mortar segment-to-segment frictional contact method for large deformations. Comput Method Appl Mech Eng 193:4891-4913. http://dx.doi.org/10.1016/j.cma.2004. 06.001 .

23. Temizer I, Wriggers P, Hughes TJR (2012) Three-dimensional mortar-based frictional contact treatment in isogeometric analysis with NURBS. Comput Method Appl Mech Eng 209-212:115-128. http://dx.doi.org/10.1016/j.cma.2011.10.014.

24. Alart P, Curnier A (1991) A mixed formulation for frictional contact problems prone to Newton like solution methods. Comput Methods Appl Mech Engrg 92:353-375. http://dx.doi.org/10.1016/00457825(91)90022-X.

25. Menezes LF, Neto DM, Oliveira MC, Alves JL (2011) Improving computational performance through HPC techniques: case study using DD3IMP in-house code. AIP Conf Proc 1353:1220-1225. http://dx.doi.org/10.1063/1.3589683.

26. Oliveira MC, Alves JL, Menezes LF (2003) Improvement of a frictional contact algorithm for strongly curved contact problems. Int J Numer Methods Eng 58:2083-2101. http://dx.doi.org/10. 1002/nme.845.

27. Piegl L, Tiller W (1997) The NURBS book. Springer, New York

28. Farin G (1997) Curves and surfaces for computer-aided geometric design: a practical guide. Academic, London

29. Neto DM, Oliveira MC, Menezes LF, Alves JL (2014). Applying Nagata patches to smooth discretized surfaces used in 3D frictional contact problems. Comput Method Appl Mech Eng 271:296-320. http://dx.doi.org/10.1016/j.cma.2013.12.008.

30. Nagata $T$ (2005) Simple local interpolation of surfaces using normal vectors. Comput Aided Geom Des 22:327-347. http://dx.doi.org/10. 1016/j.cagd.2005.01.004.

31. Neto DM, Oliveira MC, Menezes LF, Alves JL (2013) Nagata patch interpolation using surface normal vectors evaluated from the IGES file. Finite Elem Anal Des 72:35-46. http://dx.doi.org/10.1016/j. finel.2013.03.004.

32. Neto DM, Oliveira MC, Menezes LF, Alves JL (2013) Improving Nagata patch interpolation applied for tool surface description in sheet metal forming simulation. Comput-Aided Des 45:639-656. http://dx.doi.org/10.1016/j.cad.2012.10.046.

33. Zhong ZH, Nilsson L (1989) A contact searching algorithm for general contact problems. Comput Struct 33:197-209. http://dx.doi. org/10.1016/0045-7949(89)90141-7.

34. Benson DJ, Hallquist JO (1990) A single surface contact algorithm for the post-buckling analysis of shell structures. Comput Methods Appl Mech Engrg 78:141-163. http://dx.doi.org/10.1016/00457825(90)90098-7.

35. Yastrebov VA, Cailletaud G, Feyel F (2011) A local contact detection technique for very large contact and self-contact problems: sequential and parallel implementations. Trends in computational contact mechanics, LNACM, 58:227-251. http://dx.doi.org/10.1007/978-3642-22167-5 13.

36. Wriggers P (1995) Finite element algorithms for contact problems. Arch Comput Method Eng 2:1-49. http://dx.doi.org/10.1007/ BF02736195.

37. Konyukhov A, Schweizerhof K (2008) On the solvability of closest point projection procedures in contact analysis: analysis and solution strategy for surfaces of arbitrary geometry. Comput Methods Appl Mech Engrg 197:3045-3056. http://dx.doi.org/10.1016/j.cma.2008. 02.009 .

38. Heege A, Alart P (1996) A frictional contact element for strongly curved contact problems. Int J Numer Methods Engrg 39:165-184. http://dx.doi.org/10.1002/(SICI)1097-0207(19960115) 39:1<165::AID-NME846>3.0.CO;2-Y doi:10.1002/(SICI)10970207(19960115)39:1\%3c165::AID-NME846\%3e3.0.CO;2-Y.

39. Yang DY, Oh SI, Huh H, Kim YH (2002) Benchmark test B: unconstrained cylindrical bending. In: Yang DY, Oh SI, Huh H, Kim YH (eds) Proceedings of the 5th International Conference and 
Workshop on Numerical Simulation of 3D Sheet Forming Processes, vol 2. Jeju Island, South Korea, pp 757-811

40. Gelin JC, Picart P (1999) Benchmark C: reverse deep drawing of a cylindrical cup. In: Gelin JC, Picart P (eds) Proceedings of Numisheet'99. Besançon, France, pp 871-932

41. Oliveira MC, Menezes LF (2004) Automatic correction of the time step in implicit simulations of the stamping process. Finite Elem Anal Des 40:1995-2010. http://dx.doi.org/10.1016/j.finel.2004.01.009.

42. Yamada Y, Yoshimura N (1968) Plastic stress-strain matrix and its application for the solution of elastic-plastic problems by the finite element method. Int J Mech Sci 10:343-354. http://dx.doi.org/10. 1016/0020-7403(68)90001-5.

43. Thuillier S, Manach PY, Menezes LF, Oliveira MC (2002) Experimental and numerical study of reverse re-drawing of anisotropic sheet metals. J Mater Process Technol 125-126:764-771. http://dx.doi.org/10.1016/S0924-0136(02)00387-4.

44. Neto DM, Oliveira MC, Alves JL, Menezes LF (2014) Influence of the plastic anisotropy modelling in the reverse deep drawing process simulation. Mater Des 60:368-379. http://dx.doi.org/ 10.1016/j.matdes.2014.04.008. 